\title{
Anomalous Hall effect in semiconductor quantum wells in proximity to chiral $p$-wave superconductors
}

\author{
F. Yang, T. Yu, and M. W. Wv \\ Hefei National Laboratory for Physical Sciences at Microscale, Department of Physics, \\ and CAS Key Laboratory of Strongly-Coupled Quantum Matter Physics, \\ University of Science and Technology of China, Hefei, Anhui, 230026, China
}

(Dated: July 15, 2021)

\begin{abstract}
By using the gauge-invariant optical Bloch equation, we perform a microscopic kinetic investigation on the anomalous Hall effect in chiral $p$-wave superconducting states. Specifically, the intrinsic anomalous Hall conductivity in the absence of the magnetic field is zero as a consequence of Galilean invariance in our description. As for the extrinsic channel, a finite anomalous Hall current is obtained from the impurity scattering with the optically excited normal quasiparticle current even at zero temperature. From our kinetic description, it can be clearly seen that the excited normal quasiparticle current is due to an induced center-of-mass momentum of Cooper pairs through the acceleration driven by ac electric field. For the induced anomalous Hall current, we show that the conventional skew-scattering channel in the linear response makes the dominant contribution in the strong impurity interaction. In this case, our kinetic description as a supplementary viewpoint mostly confirms the results of Kubo formalism in the literature. Nevertheless, in the weak impurity interaction, this skew-scattering channel becomes marginal and we reveal that a novel induction channel from the Born contribution dominates the anomalous Hall current. This novel channel, which has long been overlooked in the literature, is due to the particle-hole asymmetry by nonlinear optical excitation. Finally, we study the case in the chiral $p$-wave superconducting state with a transverse conical magnetization, which breaks the Galilean invariance. In this situation, the intrinsic anomalous Hall conductivity is no longer zero. Comparison of this intrinsic channel with the extrinsic one from impurity scattering is addressed.
\end{abstract}

PACS numbers: 74.25.Fy, 74.25.N, 74.70.Pq, 73.21.Fg

\section{INTRODUCTION}

As one of the leading candidates for the superconducting materials with spontaneous time-reversal symmetry (TRS) breaking, $\mathrm{Sr}_{2} \mathrm{RuO}_{4}$ has attracted much attention

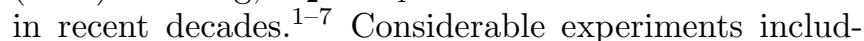
ing the neutron scattering ${ }^{8}$ and spin susceptibility $\underline{\underline{9}} \underline{-11}$ suggest that the Cooper pairing in $\mathrm{Sr}_{2} \mathrm{RuO}_{4}$ is in the spin-triplet state. The sensitive superconducting transition temperature to impurities $\frac{12,13}{13}$ and Josephson junction experiments $\underline{14}-16$ are believed to reveal the odd parity of the order parameter. Furthermore, early muon spin resonance experiment $\frac{17}{17}$ suggests that the TRS in superconducting $\mathrm{Sr}_{2} \mathrm{RuO}_{4}$ is spontaneously broken. The most convincing indication comes from the later observed polar Kerr effect in the absence of the magnetic field. $\frac{18}{}$ Particularly, the Kerr angle vanishes above the superconducting transition temperature. This clearly shows that the TRS breaking in $\mathrm{Sr}_{2} \mathrm{RuO}_{4}$ is related to the superconductivity. These results, together with the energetic consideration $\frac{6,19,20}{2}$ and symmetry analysis,$\frac{3,21,22}{,}$ reveal a chiral $p$-wave superconducting state in $\mathrm{Sr}_{2} \mathrm{RuO}_{4}$.

Although the experimental observation of the Kerr effect in superconducting $\mathrm{Sr}_{2} \mathrm{RuO}_{4} \frac{18}{}$ is very convincing, the theory of the source for this effect is not well developed. Specifically, the polar Kerr angle is proportional to the ac anomalous Hall conductivity (AHC) $\stackrel{23}{-27}$ Conventionally, in solids with the broken TRS, the intrinsic AHC arises from the anomalous velocity and hence depends on

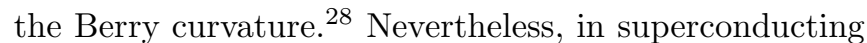
$\mathrm{Sr}_{2} \mathrm{RuO}_{4}$, even though the chiral $p$-wave character breaks TRS, for an ideal (i.e., clean single-band) model, it is theoretically revealed that the intrinsic AHC vanishes $\underline{23}-\underline{27}$ This result can be attributed to the consequence of the Galilean invariance ${ }^{29}$ Specifically, in systems with the translational symmetry, the applied ac electric field only couples to the center-of-mass (CM) momentum of the pairing electrons, independent on the relative one and hence the chiral $p$-wave character. Therefore, mechanism with broken translational symmetry for AHC induction is needed in chiral $p$-wave superconductor. After that, non-zero intrinsic $\mathrm{AHC}$ is theoretically reported by considering the inhomogeneous effect of the optical field $\stackrel{23}{=}$ Meanwhile, it is revealed that the excited collective mode,$\stackrel{24}{\underline{4}}$ which is described as a state with oscillating superconducting phase of the order parameter in time and space, $\stackrel{30-38}{-3}$ also gives finite intrinsic AHC. Nevertheless, the predicted magnitudes of AHC in these two works are much smaller than the experimentally observed one $\underline{\underline{18}}$

To date, the most promising theoretical explanation ${ }^{39}$ that unveils Kerr effect experiment in chiral p-wave superconductor involves extrinsic contribution from impurity scattering $\underline{6,25-27,40}$ Specifically, as shown in Fig. 11, with the optically excited normal Bogoliubov quasiparticle current, the anomalous Hall current can be induced from impurity scattering when the scattering probability $W_{\mathbf{k k}^{\prime}} \neq W_{\mathbf{k}^{\prime} \mathbf{k}} \stackrel{40}{ }$ Based on this picture, the theories 


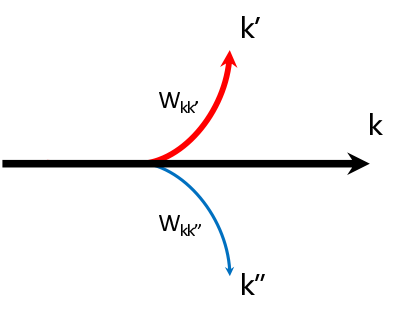

FIG. 1: (Color online) Schematic of the AHC induction from impurity scattering. In the figure, $\mathbf{k}^{\prime}=k \mathbf{e}_{\mathbf{y}}=-\mathbf{k}^{\prime \prime} ; W_{\mathbf{k k}^{\prime}}$, which only depends on the relative coordinates, denotes the scattering probability from momentum $\mathbf{k}$ to $\mathbf{k}^{\prime}$; the arrow indicates the quasiparticle current. This figure shows that with the optically excited normal Bogoliubov quasiparticle current (black arrow), the anomalous Hall current can be induced from impurity scattering when the scattering probability $W_{\mathbf{k k}^{\prime}} \neq W_{\mathbf{k k}^{\prime \prime}}=W_{\mathbf{k}^{\prime} \mathbf{k}} \stackrel{40}{ }$

of the AHC induction from impurity scattering in chiral $p$-wave superconductor in the literature are developed based on either the Kubo diagrammatic formalism ${ }^{25-27}$ or the semiclassical Boltzmann equation. $\underline{\underline{40}}$ Specifically, in Kubo diagrammatic treatment, $\stackrel{25-27}{\underline{2}}$ with the currentcurrent correlation in the linear response, finite $\mathrm{AHC}$ from impurity scattering is revealed even at zero temperature. Moreover, qualitative understanding of the AHC induction from impurity scattering is provided. Non-zero AHC induction from the conventional Born contribution in impurity scattering needs the particle-hole asymmetry, which can be induced from energy bandstructure, $\stackrel{25,26}{2}$ With the usual particle-hole symmetry, the dominant contribution to AHC comes from the skew scattering (third order of perturbation) $\stackrel{25,26}{2}$ Very recently, it is reported that the diffractive skew scattering 27 (fourth order of perturbation) also plays an important role for the AHC. As for the semiclassical approach in the chiral $p$ wave superconductor, by using the scattering $T$-matrix method, Li et al. reported the non-zero AHC based on the Boltzmann equation of the Bogoliubov quasiparticle from an intuitive viewpoint $\underline{\underline{40}}$

However, there exists obvious inconsistency between the semiclassical approach used in Ref. 40 and the experiments for the Kerr effect in $\mathrm{Sr}_{2} \mathrm{RuO}_{4} \stackrel{18}{\underline{18}}$ Specifically, the excited normal quasiparticle current in Ref. 40 is induced by the drive field of the quasiparticles from the ac electric field and hence becomes negligible around zero temperature, since the quasiparticle density decreases exponentially with temperature. Therefore, the induced AHC from impurity scattering in their work only dominates in the immediate vicinity of the superconducting transition temperature and vanishes at zero temperature. This conclusion is inconsistent with the Kerr rotation experiments where the Kerr angle achieve its maximum around zero temperature, $\stackrel{18}{=}$ and also disagrees with the prediction from Kubo diagrammatic formalism. $\underline{25}-\underline{27}$ The inconsistency lies in the fact that the acceleration of the $\mathrm{CM}$ momentum of the Cooper pairs driven by ac electric field is neglected in Ref. 40 and hence no CM momentum is excited in their work. Nevertheless, in the Kubo diagrammatic treatment, the $\mathrm{CM}$ momentum, related with the vector potential $\mathbf{A}$, is involved in the current-current correlation. Moreover, the induction of the CM momentum by ac electric field is also revealed in the conventional $s$-wave superconductors from our microscopic kinetic description $\underline{\underline{41}}$ It is established in the $s$-wave superconductor that the induction of a large CM momentum can excite Bogoliubov quasiparticles by breaking the Cooper pairs, i.e., forming the blocking region with the markedly suppressed anomalous correlation, $\underline{42-50}$ leading to the induced quasiparticle current even at zero temperature. This quasiparticle-current excitation through inducing the CM momentum is independent on the pairing symmetry ${ }^{49}$ and hence can also be expected in the chiral $p$-wave superconductor. However, this acceleration effect and its further influence on the AHC are absent in the semiclassical approach used in Ref. 40.

Furthermore, the quasiparticle correlation is overlooked in the Boltzmann equation used in Ref. 40. Nevertheless, in the $s$-wave superconductors, the quasiparticle correlation has been shown to play an important role in the optical response from the approach of the Liouville equation $\underline{51-53}$ or Bloch equation $\underline{\underline{54}-59}$ Particularly, the optically excited quasiparticle correlation breaks the particle-hole symmetry. Consequently, in the chiral $p$ wave superconducting state, one can expect the important role of the excited quasiparticle correlation in the $\mathrm{AHC}$ induction from the impurity scattering as mentioned above. However, direct extension of the Liouville equation ${ }^{51}-\underline{53}$ or Bloch equation in the chiral $p$ wave superconductors will lead to unphysical conclusions. Specifically, these equations only involve the nonlinear term $\mathbf{A}^{2}$, which leads to the pump of the quasiparticle correlation (pump effect), but the linear effect, i.e., drive effect of particles, is absent. Hence, unreasonable conclusion with zero quasiparticle current is immediately obtained. Moreover, since the pump effect is isotropic in the momentum space, the elastic scattering is ineffective.

Very recently, Yu and $\mathrm{Wu}$ pointed out $\underline{41,60}$ that the gauge invariance $31,37,61,62$ in the Liouville equation or Bloch equation in the literature ${ }^{51-59}$ is absent. Moreover, by restoring the gauge structure revealed by Nambu, $\stackrel{61,62}{,}$ they constructed the gauge-invariant optical Bloch equation for the $s$-wave superconducting state, $\stackrel{41}{\Perp}$ in which both the drive effect and the previous pump effect are included. Moreover, the quasiparticle induction by accelerating the CM momentum of Cooper pairs mentioned above is also involved in their description, and is reported to play a key role in the optical response $\underline{41,60}$ Consequently, it is natural to extend this gauge-invariant optical Bloch equation from the $s$-wave superconducting state to the chiral $p$-wave state, and use it to elucidate the fundamental nature of the AHC induction from impurity scattering.

However, the complex bandstructure and unclear interaction in $\mathrm{Sr}_{2} \mathrm{RuO}_{4}$ make a detailed calculation more 
difficult. Recently, it is theoretically predicted ${ }^{63}-65$ and preliminarily realized from the experiments $66-69$ that the chiral $p$-wave superconductivity in $\mathrm{Sr}_{2} \mathrm{RuO}_{4}$ can penetrate into the normal materials through the proximity effect. Specifically, the proximity-induced chiral $p$-wave superconductivity is theoretically reported to remain longrange even in the disordered metals ${ }^{65}$ After that, direct penetration of the superconductivity from $\mathrm{Sr}_{2} \mathrm{RuO}_{4}$ into the normal metal through a ferromagnet is observed in $\mathrm{Au} / \mathrm{SrRuO}_{3} / \mathrm{Sr}_{2} \mathrm{RuO}_{4}$ junctions $\stackrel{69}{\underline{69}}$ Naturally, proximity effect of chiral $p$-wave superconductivity in semiconductor quantum wells (QWs) is expected following the success on the realization of $s$-wave superconductivity in the semiconductor QWs via the proximity effect $\underline{\underline{70}} \underline{\underline{74}}$ Moreover, compared to the film of metals, the semiconductor QW possesses the simple Fermi surface and can be syn-

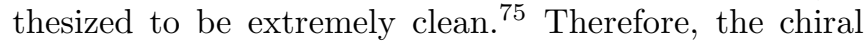
$p$-wave superconducting QWs provide an ideal platform to study the AHC induction. Moreover, the predictions revealed in superconducting QWs can still shed light on the AHC induction in $\mathrm{Sr}_{2} \mathrm{RuO}_{4}$.

In this work, with the impurity scattering included, by extending the gauge-invariant optical Bloch equation in Ref. 41 from an $s$-wave superconducting state into the chiral $p$-wave state, we systematically investigate the AHC induction in chiral $p$-wave superconducting QWs. In contrast to the well established Kubo formalism,, $25-27$ we provide a microscopic kinetic description for the $\mathrm{AHC}$ induction and reveal a new AHC induction channel from impurity scattering in our work. Specifically, we first demonstrate that the intrinsic $\mathrm{AHC}$ without magnetic field is zero as a consequence of the Galilean invariance in our description, in agreement with the previous works: $\stackrel{23}{\underline{2}-27}$ As for the extrinsic AHC, we show that even at zero temperature, there exists optically excited nonzero normal quasiparticle current, and a finite anomalous Hall current from impurity scattering (Fig. 1) is obtained, in consistence with the experiment $\underline{18}$ We further reveal that the excited quasiparticle current around zero temperature arises from the induced CM momentum of Cooper pairs through the acceleration driven by the ac electric field, which has been overlooked in the previous semiclassical approach $\underline{40}$ As mentioned above, the induction of a large CM momentum can excite Bogoliubov quasiparticles by breaking the Cooper pairs, i.e., forming the blocking region with markedly suppressed anomalous correlation, $\stackrel{42-50}{=}$ leading to the induced quasiparticle current and hence AHC from impurity scattering even at zero temperature.

Moreover, we find that there exist two AHC induction channels from the impurity scattering: Channel I, conventional linear channel, where the AHC is induced from the skew scattering in the linear response; Channel II, anomalous nonlinear channel, where the AHC is induced from the Born contribution due to the broken particle-hole symmetry by the nonlinearly excited quasiparticle correlation, as mentioned above. Particularly, we point out that Channel I from our microscopic ki- netic description, as a supplementary viewpoint, mostly confirms the results of Kubo diagrammatic formalism of the skew scattering. However, we show that this conventional linear channel (Channel I) only dominates in the strong impurity interaction, whereas in the weak impurity interaction, the anomalous nonlinear channel (Channel II) makes the dominant contribution. Consequently, Channel II may also play an important role in $\mathrm{Sr}_{2} \mathrm{RuO}_{4}$, since $\mathrm{Sr}_{2} \mathrm{RuO}_{4}$ is essentially in the weak impurity scattering limit in order to achieve the superconducting phase , $, 6,12,13^{2}$ Nevertheless, to the best of our knowledge, Channel II has long been overlooked in the literature, due to the difficulty in treating the quasiparticle correlation in the previous semiclassical approach ${ }^{40}$ or including the nonlinear effect in Kubo diagrammatic formalism ${ }^{25-27}$

Furthermore, motivated by the recent observed penetration of the superconductivity from $\mathrm{Sr}_{2} \mathrm{RuO}_{4}$ into the normal metal through a ferromagnet in metal/ferromagnet/ $\mathrm{Sr}_{2} \mathrm{RuO}_{4}$ junctions, ${ }^{69}$ we also study the AHC induction in chiral $p$-wave superconducting QWs in the presence of a magnetization. In our work, we consider a specific transverse conical magnetization, $\underline{\underline{76}} \underline{\underline{80}}$ which breaks the translational symmetry and hence the Galilean invariance. In this situation, the intrinsic AHC, attributed from the anomalous velocity, is no longer zero. Comparison of this intrinsic AHC with the extrinsic one from impurity scattering is also addressed in our work.

Finally, we also investigate the optical response of the chiral $p$-wave superconducting order parameter. Compared to the situation in the $s$-wave superconducting state, $\stackrel{41}{\longleftarrow}$ unique optical excitation for the triplet-vector ${ }^{81}$ orientation and new feature in the collective-mode $30-38$ excitation are presented in our work.

This paper is organized as follows. In Sec. II, we introduce our model. The analytic analysis and specific numerical results for the AHC induction are presented in Sec. III and IV, respectively. We summarize in Sec. IV.

\section{MODEL}

In this section, we present the Hamiltonian and set up the gauge-invariant optical Bloch equation for the chiral $p$-wave superconducting state by following the previous work for the $s$-wave case $\underline{\underline{41}}$

\section{A. Hamiltonian}

The free Bogoliubov-de Gennes (BdG) Hamiltonian of the chiral $p$-wave superconducting QWs is given by

$H_{0}=\int \frac{d \mathbf{r}}{2} \Psi^{\dagger}\left\{\left[\xi_{\mathbf{k}-e \mathbf{A}(x) \tau_{3}}+e \phi(x)+\mathbf{h}_{\mathbf{r}} \cdot \tilde{\boldsymbol{\sigma}}\right] \tau_{3}+\hat{\Delta}_{\mathbf{k}}^{\mathbf{r}}\left(\mathbf{d}_{\mathbf{0}}\right)\right\} \Psi$, 
with

$\hat{\Delta}_{\mathbf{k}}^{\mathbf{r}}\left(\mathbf{d}_{\mathbf{0}}\right)=\frac{\left(\mathbf{d}_{0} \cdot \boldsymbol{\sigma}\right) i \sigma_{2}}{2}\left(\left\{e^{i \eta \theta_{\mathbf{k}}}, e^{i \psi(\mathbf{r})}\right\} \tau_{+}-\left\{e^{-i \eta \theta_{\mathbf{k}}}, e^{-i \psi(\mathbf{r})}\right\} \tau_{-}\right)$.

Here, $\Psi=\left(\Psi_{\uparrow}(x), \Psi_{\downarrow}(x), \Psi_{\uparrow}^{\dagger}(x), \Psi_{\downarrow}^{\dagger}(x)\right)^{T}$ is the Nambu spinors with $x=(t, \mathbf{r})$ being the time-space point; $\xi_{k}=$ $\varepsilon_{k}-\mu$ and $\varepsilon_{k}=\frac{k^{2}}{2 m}$ with $m$ and $\mu$ being the effective mass and chemical potential; $\mathbf{A}(x)$ and $\phi(x)$ represent the vector and scalar potential, respectively; $\mathbf{d}_{0}=\Delta_{0} \mathbf{e}_{\mathbf{z}}$ is defined as the triplet vector; ${ }^{81} \Delta_{0}$ and $\psi(x)$ stand for the magnitude and superconducting phase of the order parameter, respectively; $\eta= \pm 1$ is the chiral character; $\{$,$\} denotes the anticommutator; \mathbf{h}_{\mathbf{r}}$ represents the magnetization with spatial dependence; $\tilde{\boldsymbol{\sigma}}=\operatorname{diag}\left(\boldsymbol{\sigma}, \boldsymbol{\sigma}^{*}\right) ; \sigma_{i}$ and $\tau_{i}$ are the Pauli matrices in spin and particle-hole spaces, respectively.

It is first revealed by Nambu that under a gauge transformation $\Psi(x) \rightarrow e^{i \tau_{3} \chi(x)} \Psi(x)$, to restore the gauge invariance of the BdG Hamiltonian, the vector potential, scalar potential, and superconducting phase must transform as 61,62

$$
\begin{aligned}
e A_{\mu} & \rightarrow e A_{\mu}-\partial_{\mu} \chi(x), \\
\psi(x) & \rightarrow \psi(x)+2 \chi(x)
\end{aligned}
$$

where the four vectors are $A_{\mu}=(\phi, \mathbf{A})$ and $\partial_{\mu}=$ $\left(\partial_{t},-\nabla\right)$.

\section{B. Optical Bloch equation}

Following the previous work for the $s$-wave superconducting state, $\stackrel{41}{=}$ by introducing the Wilson line ${ }^{82-86}$ to retain the gauge invariance, via the nonequilibrium Green function method in the quasiparticle approximation, ${ }^{83,85,87}$ we set up the gauge-invariant optical Bloch equations for the chiral $p$-wave superconducting state (refer to Appendix A ) and choose a specific gauge with zero superconducting phase for the convenience of the physical analysis. The optical Bloch equations read:

$$
\begin{aligned}
& \partial_{T} \rho_{\mathbf{k}}+i\left[\left(\xi_{k}+\mu_{\mathrm{eff}}+\mathbf{h}_{\mathbf{R}} \cdot \tilde{\boldsymbol{\sigma}}+\frac{\mathbf{p}_{\mathbf{s}}{ }^{2}}{2 m}\right) \tau_{3}+\hat{\Delta}_{\mathbf{k}}(\mathbf{d}), \rho_{\mathbf{k}}\right] \\
& +\left\{\frac{\partial_{t} \mathbf{p}_{\mathbf{s}}+\boldsymbol{\nabla}_{\mathbf{R}}\left(\mathbf{h}_{\mathbf{R}} \cdot \tilde{\boldsymbol{\sigma}}\right)}{2} \tau_{3}, \partial_{\mathbf{k}} \rho_{\mathbf{k}}\right\}-\left[\frac{\boldsymbol{\nabla}_{\mathbf{R}} \cdot \mathbf{p}_{\mathbf{s}}}{4 m} \tau_{3}, \tau_{3} \rho_{\mathbf{k}}\right] \\
& -\left[\frac{\mathbf{p}_{\mathbf{s}}}{2 m} \tau_{3}, \tau_{3} \boldsymbol{\nabla}_{\mathbf{R}} \rho_{\mathbf{k}}\right]-\left[\frac{i}{8 m} \tau_{3}, \boldsymbol{\nabla}_{\mathbf{R}}^{2} \rho_{\mathbf{k}}\right]=\left.\partial_{t} \rho_{\mathbf{k}}\right|_{\text {scat }}, \quad(5)
\end{aligned}
$$

where $\rho_{\mathbf{k}}$ is the density matrix in the Nambu $\otimes$ spin space and $\left.\partial_{t} \rho_{\mathbf{k}}\right|_{\text {scat }}$ represents scattering terms due to the electron-impurity scattering; [, ] stands for the commutator.

In Eq. (5), both the superconducting momentum $\mathbf{p}_{s}=$ $-e \mathbf{A}+\nabla_{\mathbf{R}} \psi$ and the effective chemical potential $\mu_{\mathrm{eff}}=$ $e \phi+\partial_{t} \psi$ are gauge-invariant physical quantities. $37,61,88$ They are related by the acceleration relation: $:^{37,61,88}$

$$
\partial_{t} \mathbf{p}_{s}=\nabla_{\mathbf{R}} \mu_{\mathrm{eff}}+e \mathbf{E} .
$$

The superconducting order parameter in Eq. (5) is written as

$$
\hat{\Delta}_{\mathbf{k}}(\mathbf{d})=(\mathbf{d} \cdot \boldsymbol{\sigma})\left(e^{i \eta \theta_{\mathbf{k}}} \tau_{+}-e^{-i \eta \theta_{\mathbf{k}}} \tau_{-}\right) i \sigma_{2},
$$

with $\mathbf{d}=\mathbf{d}_{\mathbf{0}}+\delta \mathbf{d}_{\mathbf{k}}^{\mathrm{HF}}$ being the effective triplet vector and $\delta \mathbf{d}_{\mathbf{k}}^{\mathrm{HF}}=\operatorname{Tr}\left[H_{\mathbf{k}}^{\mathrm{HF}} \tau_{-} i \sigma_{2}^{*} \boldsymbol{\sigma} / 2\right] e^{-i \eta \theta_{\mathbf{k}}}$ standing for the fluctuation of the triplet vector from the Hartree-Fock (HF) self-energy $H_{\mathbf{k}}^{\mathrm{HF}}=\sum_{\mathbf{k}^{\prime}} V_{\mathbf{k}-\mathbf{k}^{\prime}} \tau_{3}\left(\rho_{\mathbf{k}^{\prime}}-\rho_{\mathbf{k}^{\prime}}^{0}\right) \tau_{3}$ due to the Coulomb interaction. ${ }^{41}$ Here, $V_{\mathbf{q}}=2 \pi e^{2} /\left(q \epsilon_{q}\right)$ is the $2 \mathrm{D}$ screened Coulomb potential; $\epsilon_{q}=\epsilon_{0} \kappa_{0}(1+\kappa / q)$ with $\epsilon_{0}$ and $\kappa_{0}$ representing the vacuum permittivity and relative dielectric constant; the screening constant is given by $\kappa=2 m e^{2} /\left(\epsilon_{0} \kappa_{0}\right)$ at low temperature. From Eq. (77), one can obtain the optical response of the order parameter.

The charge-neutrality $\underline{40,41,60,89} \underline{\underline{91}}$ condition requires

$$
n=\sum_{\mathbf{k}}\left\{1-\frac{\xi_{\mathbf{k}}+\mu_{\mathrm{eff}}}{\sqrt{\left(\xi_{\mathbf{k}}+\mu_{\mathrm{eff}}\right)^{2}+\Delta_{0}^{2}}}+\operatorname{Tr}\left(\delta \rho_{\mathbf{k}}^{q} t_{3}\right)\right\},
$$

with $t_{3}=U_{\mathbf{k}}^{\dagger} \tau_{3} U_{\mathbf{k}}$ and $\delta \rho_{\mathbf{k}}^{q}=\rho_{\mathbf{k}}^{q}-\rho_{\mathbf{k}}^{q, 0} \cdot \rho_{\mathbf{k}}^{q}=U_{\mathbf{k}}^{\dagger} \rho_{\mathbf{k}} U_{\mathbf{k}}$ is the density matrix in the quasiparticle space and $n$ stands for the total electron density. $U_{\mathbf{k}}$ represents the unitary transformation matrix from the particle space to the quasiparticle one, which is written as

$$
U_{\mathbf{k}}=\left(\begin{array}{cc}
u_{\mathbf{k}} e^{i \eta \theta_{\mathbf{k}} / 2} & v_{\mathbf{k}} e^{i \eta \theta_{\mathbf{k}} / 2} \sigma_{1} \\
-v_{\mathbf{k}} e^{-i \eta \theta_{\mathbf{k}} / 2} \sigma_{1} & u_{\mathbf{k}} e^{-i \eta \theta_{\mathbf{k}} / 2}
\end{array}\right)
$$

with $u_{\mathbf{k}}=\sqrt{\frac{1}{2}+\frac{\xi_{\mathbf{k}}}{2 E_{\mathbf{k}}}}$ and $v_{\mathbf{k}}=\sqrt{\frac{1}{2}-\frac{\xi_{\mathbf{k}}}{2 E_{\mathbf{k}}}} . \quad E_{k}=$ $\sqrt{\xi_{k}^{2}+\Delta_{0}^{2}}$ denotes the quasiparticle energy spectra. From Eq. (8), the optical response of the effective chemical potential $\mu_{\text {eff }}$ can be obtained to keep the charge neutrality. $40,41,60$

The equilibrium state $\rho_{\mathbf{k}}^{0}\left(\rho_{\mathbf{k}}^{q, 0}\right)$ in the particle (quasiparticle) space is given by $\rho_{\mathbf{k}}^{0}=U_{\mathbf{k}} \rho_{\mathbf{k}}^{q, 0} U_{\mathbf{k}}^{\dagger}$ with

$$
\rho_{\mathbf{k}}^{q, 0}=\left(1-\tau_{3}\right) / 2+f\left(E_{k}\right) \tau_{3} .
$$

Here, $f(x)$ is the Fermi distribution.

As for the scattering term, we mainly consider the long-range electron-impurity scattering: 87

$$
\begin{aligned}
& \left.\partial_{t} \rho_{\mathbf{k}}\right|_{\text {scat }}=-\pi n_{i} \sum_{\mathbf{k}^{\prime}} \sum_{\lambda=1}^{4} \delta\left(E_{k^{\prime} \lambda}-E_{k \lambda}\right) \\
& \times\left(T_{\mathbf{k k}^{\prime}} \Gamma_{\mathbf{k}^{\prime}}^{\lambda} T_{\mathbf{k}^{\prime} \mathbf{k}} \Gamma_{\mathbf{k}}^{\lambda} \rho_{\mathbf{k}}-T_{\mathbf{k k}^{\prime}} \rho_{\mathbf{k}^{\prime}} \Gamma_{\mathbf{k}^{\prime}}^{\lambda} T_{\mathbf{k}^{\prime} \mathbf{k}} \Gamma_{\mathbf{k}}^{\lambda}+\text { H.c. }\right) .
\end{aligned}
$$

Here, $n_{i}$ is the impurity density; $E_{\mathbf{k} \lambda}=E_{\mathbf{k}} \delta_{\lambda, 1}+E_{\mathbf{k}} \delta_{\lambda, 2}-$ $E_{\mathbf{k}} \delta_{\lambda, 3}-E_{\mathbf{k}} \delta_{\lambda, 4} ; \Gamma_{\mathbf{k}}^{\lambda}=U_{\mathbf{k}} Q^{\lambda} U_{\mathbf{k}}^{\dagger}$ denote the projection operators with $Q^{\lambda}=\operatorname{diag}\left(\delta_{\lambda, 1}, \delta_{\lambda, 2}, \delta_{\lambda, 3}, \delta_{\lambda, 4}\right)$; the scattering $T$-matrix $T_{\mathbf{k k}^{\prime}}$ reads (refer to Appendix $\mathrm{B}$ ):

$$
T_{\mathbf{k k}^{\prime}}(E)=\frac{z_{i} V_{\mathbf{k}-\mathbf{k}^{\prime}} \tau_{3}}{1+i \tau_{3} \pi \nu z_{i} V_{0} E / \sqrt{E^{2}-\Delta_{0}^{2}}},
$$

with $\nu$ and $z_{i}$ being the density of states and electronimpurity interaction strength, respectively. Tuning $z_{i}$ 
can model different scatterings including the short-range scattering. Moreover, the prediction revealed in our work can help understanding the AHC induction in disordered superconducting $\mathrm{Sr}_{2} \mathrm{RuO}_{4}$ samples with different impurity potentials. Following the standard treatment of energy $E$ in the scattering $T$-matrix $T_{\mathbf{k k}^{\prime}}(E)$ for normal state $\left(E=E_{F}\right), \underline{92-97}$ in our work, by considering the elastic scattering, we takes the energy $E=E_{k}$ in the $T$-matrix $T_{\mathbf{k k}^{\prime}}(E)$, exactly same as the previous work in superconducting state. $\stackrel{40}{ }$ Then, Eq. (12) becomes $T_{\mathbf{k k}^{\prime}}=$ $M_{\mathbf{k k}^{\prime}} e^{i \delta_{k} \tau_{3}} \tau_{3}$ with amplitude $M_{\mathbf{k k}^{\prime}}=z_{i} V_{\mathbf{k}-\mathbf{k}^{\prime}}\left|\cos \delta_{k}\right|$ and phase $\delta_{k}=-\arctan \left(z_{i} \pi \nu V_{0} E_{\mathbf{k}} /\left|\xi_{\mathbf{k}}\right|\right)$.

Finally, as mentioned in the introduction, with the gauge-invariant optical Bloch equations [Eq. (5)], the drive effect of the particles from the drive field $\partial_{t} \mathbf{p}_{\mathbf{s}}$, which excites the quasiparticle population in the linear response, and the pump effect from $p_{s}^{2} /(2 m)$ term, which induces the quasiparticle correlation from the nonlinear effect, are both kept. Moreover, besides the optical field, the SG force $\boldsymbol{\nabla}_{\mathbf{R}}\left(\mathbf{h}_{\mathbf{R}} \cdot \tilde{\boldsymbol{\sigma}}\right)$ also provides a drive field to drive the particles.

The current during the optical response is given by:

$$
\mathbf{I}=\frac{e}{2 m} \int \frac{d \mathbf{k}}{(2 \pi)^{2}} \operatorname{Tr}\left(\mathbf{k} \rho_{\mathbf{k}}\right)
$$

\section{ANALYTIC ANALYSIS}

In this section, from the extended optical Bloch equations [Eq. (5)], we analytically show that the intrinsic $\mathrm{AHC}$ without magnetic field is zero as a consequence of the Galilean invariance, in agreement with the previous works ${ }^{23-27}$ As for the extrinsic channel, we show that the normal quasiparticle current is excited even at zero temperature, and then, the $\mathrm{AHC}$ is induced from impurity scattering. It is further revealed that the excited normal quasiparticle current arises from the induced CM momentum of Cooper pairs through the acceleration driven by the ac electric field, which has been overlooked in the previous semiclassical approach. 40

Moreover, two AHC induction channels from the impurity scattering are analytically revealed: Channel I, conventional linear channel, where the AHC is induced from the skew scattering in the linear response; Channel II, anomalous nonlinear channel, where the AHC is induced from the Born contribution due to the broken particlehole symmetry by the nonlinearly excited quasiparticle correlation. Particularly, we point out that Channel I from our microscopic kinetic description mostly confirms the results of Kubo diagrammatic formalism of the skew scattering. 25,26 Nevertheless, to the best of our knowledge, Channel II has long been overlooked in the literature, due to the difficulty in treating the quasiparticle correlation in the previous semiclassical approach 40 or including the nonlinear effect in Kubo diagrammatic formalism. ${ }^{25-27}$

\section{A. Zero intrinsic AHC due to Galilean invariance}

We first analytically show that in the absence of the magnetic field, the intrinsic $\mathrm{AHC}$ for the chiral $p$-wave superconducting state is zero as a consequence of the Galilean invariance. Specifically, without the magnetic field and impurity scattering included, under a unitary transformation $P_{\mathbf{k}}=e^{i \eta \theta_{\mathbf{k}} / 2 \tau_{3}}$, Eq. (15) is transformed into:

$$
\begin{aligned}
& \partial_{T} \rho_{\mathbf{k}}^{s}+i\left[\left(\begin{array}{cc}
\xi_{\mathbf{k}}+\mu_{\mathrm{eff}}+\frac{\mathbf{p}_{\mathbf{s}}^{2}}{2 m} & (\mathbf{d} \cdot \boldsymbol{\sigma}) i \sigma_{2} \\
(\mathbf{d} \cdot \boldsymbol{\sigma}) i \sigma_{2}^{*} & -\xi_{\mathbf{k}}-\mu_{\mathrm{eff}}-\frac{\mathbf{p}_{\mathbf{s}}^{2}}{2 m}
\end{array}\right), \rho_{\mathbf{k}}^{s}\right] \\
& +\left\{\frac{\partial_{t} \mathbf{p}_{\mathbf{s}}}{2} \tau_{3}, \partial_{\mathbf{k}} \rho_{\mathbf{k}}^{s}\right\}-\left[\frac{\boldsymbol{\nabla}_{\mathbf{R}} \cdot \mathbf{p}_{\mathbf{s}}}{4 m} \tau_{3}, \tau_{3} \rho_{\mathbf{k}}^{s}\right]-\left[\frac{i \tau_{3}}{8 m}, \nabla_{\mathbf{R}}^{2} \rho_{\mathbf{k}}^{s}\right] \\
& -\left[\frac{\mathbf{p}_{\mathbf{s}}}{2 m} \tau_{3}, \tau_{3} \boldsymbol{\nabla}_{\mathbf{R}} \rho_{\mathbf{k}}^{s}\right]+\left\{\frac{\partial_{t} \mathbf{p}_{\mathbf{s}}}{2} \tau_{3},\left[\left(P_{\mathbf{k}}^{\dagger} \partial_{\mathbf{k}} P_{\mathbf{k}}\right), \rho_{\mathbf{k}}^{s}\right]\right\}=0
\end{aligned}
$$

with $\rho_{\mathbf{k}}^{s}=P_{\mathbf{k}}^{\dagger} \rho_{\mathbf{k}} P_{\mathbf{k}}$ and $P_{\mathbf{k}}^{\dagger} \partial_{\mathbf{k}} P_{\mathbf{k}}=i \eta /(2 k) \tau_{3} \mathbf{e}_{\boldsymbol{\theta}_{\mathbf{k}}}$.

It can be easily demonstrated that due to the fact $\left\{\tau_{3},\left[\tau_{3}, \rho_{\mathbf{k}}^{s}\right]\right\} \equiv 0$, the last term in Eq. (14), which comes from the Berry curvature, is exactly zero. Then, the optical Bloch equations [Eq. (14)] for the chiral $p$-wave superconducting state are exactly identical as that for the $s$-wave ones ${ }^{41}$ in the Nambu space. This can be attributed to the consequence of the Galilean invariance, ${ }^{29}$ since in systems with the translational symmetry, the optical field only couples to the CM momentum of the pairing electrons, independent on the relative one and hence the chiral $p$-wave character. Consequently, it is directly concluded that the intrinsic $\mathrm{AHC}$ for the chiral $p$-wave superconducting state is zero, in agreement with the previous works. ${ }^{23-27}$

\section{B. Berry curvature}

In this part, we first simplify our optical Bloch equations to demonstrate that a finite normal quasiparticle current is excited even at zero temperature. In the absence of the magnetic field, with the translational symmetry, the spatial gradient terms in Eq. (5) can be neglected. For the convenience of the physical analysis, we transform the remaining optical Bloch equations from the particle space into the quasiparticle one as:

$$
\begin{aligned}
& \partial_{T} \rho_{\mathbf{k}}^{q}+i\left[E_{k} \tau_{3}, \rho_{\mathbf{k}}^{q}\right]+\frac{1}{2}\left\{\partial_{t} \mathbf{p}_{\mathbf{s}} t_{3}, \partial_{\mathbf{k}} \rho_{\mathbf{k}}^{q}\right\} \\
& +\frac{1}{2}\left\{\partial_{t} \mathbf{p}_{\mathbf{s}} t_{3},\left[U_{\mathbf{k}}^{\dagger} \partial_{\mathbf{k}} U_{\mathbf{k}}, \rho_{\mathbf{k}}^{q}\right]\right\}=\left.\partial_{t} \rho_{\mathbf{k}}^{q}\right|_{\text {scat }} .
\end{aligned}
$$

Here, we have neglected the pump effect, which is isotropic in the momentum space and hence has no direct influence on quasiparticle current. We also neglect the HF term and effective chemical potential for simplification.

Around zero temperature with the initial quasiparticle distribution $f\left(E_{k}\right) \approx 0$ in Eq. (10), in the linear response, 
the third term in Eq. (15), which corresponds to the drive field to drive the quasiparticles, is zero and hence has no contribution to quasiparticle current. Whereas the fourth term in Eq. (15), which arises from Berry curvature, is finite. Consequently, from the fourth term in Eq. (15), by assuming $\partial_{t} \mathbf{p}_{\mathbf{s}}=e E_{0} e^{i \Omega t} \mathbf{e}_{\mathbf{x}}$ and neglecting the scattering, one has the optically excited normal quasiparticle current:

$$
\mathbf{I}_{0}=-i e^{2} E_{0} \nu \int d \epsilon_{k} \frac{\left(2 u_{k} v_{k}\right)^{2} \varepsilon_{k}}{m \Omega E_{k}} \mathbf{e}_{\mathbf{x}}
$$

which is non-zero even at zero temperature.

This can be understood as follows. The Berry curvature [fourth term in Eq. (15)], overlooked in previous semiclassical approach, $\stackrel{40}{=}$ is related to the acceleration of the CM momentum of Cooper pairs driven by the ac electric field [Eq. [6])], which excites finite CM momentum. Particularly, as mentioned in the introduction, the induction of a large CM momentum can excite Bogoliubov quasiparticles by breaking the Cooper pairs, i.e., forming the blocking region with the markedly suppressed anomalous correlation, $\stackrel{42-50}{=}$ leading to the excited quasiparticle current even at zero temperature.

\section{Extrinsic AHC from impurity scattering}

We next analytically show that with the optically excited normal quasiparticle current, the anomalous Hall current can be induced from the impurity scattering. In our study, we mainly consider the relatively weak impurity interaction $z_{i} \leq 1$. The impurity scattering in the quasiparticle space is given by:

$$
\begin{aligned}
\left.\partial_{t} \rho_{\mathbf{k}}^{q}\right|_{\text {scat }}= & -\pi n_{i} \nu \sum_{\xi_{k^{\prime}}= \pm \xi_{k}} \frac{E_{k}}{\left|\xi_{k}\right|} \int \frac{d \theta_{\mathbf{k}^{\prime}}}{2 \pi}\left[W_{\mathbf{k k}^{\prime}}\left(\rho_{\mathbf{k}}^{q}-\rho_{\mathbf{k}^{\prime}}^{q}\right)\right. \\
& \left.+Y_{\mathbf{k k}^{\prime}}\left[\tau_{3}, \rho_{\mathbf{k}^{\prime}}^{q}\right]+\text { H.c. }\right] .
\end{aligned}
$$

Here, $\quad W_{\mathbf{k k}^{\prime}}=t_{\mathbf{k k}^{\prime}} \operatorname{Tr}\left(t_{\mathbf{k}^{\prime} \mathbf{k}}\right)+Y_{\mathbf{k k}^{\prime}} \tau_{3}$ and $Y_{\mathbf{k k}^{\prime}}=$ $t_{\mathbf{k k}^{\prime}} \operatorname{Tr}\left(t_{\mathbf{k}^{\prime} \mathbf{k}} \tau_{3}\right)$ with $t_{\mathbf{k k}^{\prime}}=U_{\mathbf{k}}^{\dagger} \tau_{3} U_{\mathbf{k}^{\prime}} / 2$. By neglecting the second term in the right-hand side of Eq. (17) and assuming $\sum_{\xi_{k^{\prime}}= \pm \xi_{k}} \approx 2 \delta_{k, k^{\prime}}$ for simplification, Eq. (17) approximately becomes

$\left.\partial_{t} \rho_{\mathbf{k}}^{q}\right|_{\text {scat }}=-2 \pi n_{i} \nu \delta_{k, k^{\prime}} \frac{E_{k}}{\left|\xi_{k}\right|} \int \frac{d \theta_{\mathbf{k}^{\prime}}}{2 \pi}\left[W_{\mathbf{k k}^{\prime}}\left(\rho_{\mathbf{k}}^{q}-\rho_{\mathbf{k}^{\prime}}^{q}\right)+\right.$ H.c. $]$.

One can separate the remaining scattering probability $W_{\mathbf{k k}^{\prime}}=W_{\mathbf{k k}^{\prime}}^{s}+W_{\mathbf{k k}^{\prime}}^{a}$ into the symmetric part $W_{\mathbf{k k}^{\prime}}^{s}=$ $\left(W_{\mathbf{k k}^{\prime}}+W_{\mathbf{k}^{\prime} \mathbf{k}}\right) / 2$, which provides the momentum relaxation, and anti-symmetric one $W_{\mathbf{k k}^{\prime}}^{a}=\left(W_{\mathbf{k k}^{\prime}}-W_{\mathbf{k}^{\prime} \mathbf{k}}\right) / 2$, which breaks the TRS and can induce the AHC. Particularly, the anti-symmetric scattering probability $W_{\mathbf{k k}^{\prime}}^{a}$ at $z_{i} \leq 1$ is given by

$$
W_{\mathbf{k k}^{\prime}}^{a}=\sin \eta \delta \theta_{\mathbf{k k}^{\prime}}\left|V_{\mathbf{k k}^{\prime}}\right|^{2}\left(\begin{array}{cc}
w_{k}^{d} & w_{k}^{o} \sigma_{1} \\
w_{k}^{o} \sigma_{1} & w_{k}^{d}
\end{array}\right)
$$

including the diagonal part $w_{k}^{d} \approx 2 u_{k}^{2} v_{k}^{2} \sin 2 \delta_{k}$ and offdiagonal one $w_{k}^{o} \approx i u_{k} v_{k}$. Here, $\delta \theta_{\mathbf{k k}^{\prime}}=\theta_{\mathbf{k}}-\theta_{\mathbf{k}^{\prime}}$.

Consequently, based on the analysis in Sec. IIIB, by assuming $\partial_{t} \mathbf{p}_{\mathbf{s}}=e E_{0}\left(e^{i \Omega t}+e^{-i \Omega t}\right) \mathbf{e}_{\mathbf{x}}$ and expanding $\rho_{\mathbf{k}}^{q}=\sum_{l} \rho_{\mathbf{k}}^{q, l} e^{i l \Omega t}$ in the Fourier frequency space, the optical Bloch equations [Eq. [15)] can be approximately simplified as:

$$
\rho_{\mathbf{k}, 0}^{q, 1}=-\frac{1}{i \Omega+\tau_{p, k}^{-1}}\left[\frac{e E_{0} k_{x}\left(2 u_{k} v_{k}\right)^{2}}{m E_{k}}\left(\rho_{\mathbf{k}, 3}^{q, 0}+\rho_{\mathbf{k}, 3}^{q, 2}\right)+\Gamma_{\mathbf{k}}^{d}\right],
$$

with

$$
\begin{aligned}
& \rho_{\mathbf{k}, \pm}^{q, 2}=i \frac{e E_{0} 2 u_{k} v_{k}}{2 \Omega \pm 2 E_{k}}\left(\partial_{k_{x}} \rho_{\mathbf{k}, 0}^{q, 1}\right), \\
& \rho_{\mathbf{k}, 3}^{q, 2}=i \frac{\Gamma_{\mathbf{k}-}^{o}-\Gamma_{\mathbf{k}+}^{o}}{2 \Omega}, \\
& \Gamma_{\mathbf{k}}^{d}=w_{k}^{d} n_{i} \nu \int \frac{2 E_{k} d \theta_{\mathbf{k}^{\prime}}}{\left|\xi_{k}\right|} \sin \eta \delta \theta_{\mathbf{k k}^{\prime}}\left|V_{\mathbf{k k}^{\prime}}\right|^{2}\left(\rho_{\mathbf{k} 0}^{q, 1}-\rho_{\mathbf{k}^{\prime} 0}^{q, 1}\right) . \\
& \Gamma_{\mathbf{k} \pm}^{o}=w_{k}^{o} n_{i} \nu \int \frac{E_{k} d \theta_{\mathbf{k}^{\prime}}}{\left|\xi_{k}\right|} \sin \eta \delta \theta_{\mathbf{k k}^{\prime}}\left|V_{\mathbf{k k}^{\prime}}\right|^{2}\left(\rho_{\mathbf{k} \pm}^{q, 2}-\rho_{\mathbf{k}^{\prime} \pm}^{q, 2}\right) .
\end{aligned}
$$

Here, $\rho_{\mathbf{k}, i}^{q, l}=\operatorname{Tr}\left(\rho_{\mathbf{k}}^{q, l} \tau_{i}\right)$ and the momentum relaxation rate $\tau_{p, k}^{-1}$ is given by $\tau_{p, k}^{-1} \approx \pi n_{i} \nu \int \frac{d \theta_{\mathbf{k}^{\prime}}}{2 \pi} \frac{E_{k}}{\left|\xi_{k}\right|} \operatorname{Tr}\left(W_{\mathbf{k k}^{\prime}}^{s}\right)(1-$ $\left.\cos \delta \theta_{\mathbf{k k}^{\prime}}\right) \approx 4 \pi n_{i} \nu\left|V_{0}\right|^{2}\left(1-u_{k}^{2} v_{k}^{2}\right)$.

In the right-hand side of Eq. (20), the first term corresponds to the normal quasiparticle-current excitation from the acceleration of the CM momentum of Cooper pair, as mentioned in Sec. IIIB. The second term denotes the response of quasiparticle-current induction to the nonlinear optical excitation. The third term $\left(\Gamma_{\mathbf{k}}^{d} \propto\right.$ $\left.\left|V_{\mathbf{k k}^{\prime}}\right| \sin 2 \delta_{k} \propto z_{i}^{3}\right)$ stands for the skew scattering. Then, from Eq. (20), the anomalous Hall current is obtained as

$$
I_{y}=\frac{2 e}{m} \int \frac{d \mathbf{k}}{(2 \pi)^{2}}\left(k_{y} \rho_{\mathbf{k} 0}^{q, l=1}\right)=I_{y}^{\mathrm{I}}+I_{y}^{\mathrm{II}},
$$

with

$$
\begin{aligned}
I_{y}^{\mathrm{I}} & =-\frac{2 e}{m} \int \frac{d \mathbf{k}}{(2 \pi)^{2}} \frac{k_{y} \Gamma_{\mathbf{k}}^{d}}{i \Omega+\tau_{p, k}^{-1}}, \\
I_{y}^{\mathrm{II}} & =-i \frac{2 e^{2}}{m} E_{0} \int \frac{d \mathbf{k}}{(2 \pi)^{2}} \frac{4 u^{2} v^{2} k_{x} k_{y}}{i \Omega+\tau_{p, k}^{-1}} \frac{\Gamma_{\mathbf{k}-}^{o}-\Gamma_{\mathbf{k}+}^{o}}{2 \Omega m E_{k}} .
\end{aligned}
$$

Consequently, there exist two AHC induction channels from the impurity scattering: Channel I, in which the anomalous Hall current $I_{y}^{\mathrm{I}}$ is induced from the skew scattering $\left(\Gamma_{\mathbf{k}}^{d} \propto z_{i}^{3}\right)$ in the linear response $\left(\Gamma_{\mathbf{k}}^{d} \propto E_{0}\right)$, and hence, we referred to this channel as the conventional linear channel; Channel II, where the anomalous Hall current $I_{y}^{\mathrm{II}}$ comes from the response of quasiparticle-current induction to the nonlinear optical excitation [second term in the right-hand side of Eq. (20)], and hence, we referred to this channel as the anomalous nonlinear channel. 
For channel I, from Eq. (26), one approximately obtains

$$
I_{y}^{\mathrm{I}}=E_{0} n_{i} \eta \Delta_{0} \frac{\nu^{2} e^{2}}{2 m} \int d\left(\frac{\varepsilon_{k}}{\Delta_{0}}\right) \frac{\Delta_{0}^{4} \varepsilon_{k}}{E_{k}^{4}\left|\xi_{k}\right|} \frac{\tau_{p, k}^{2} 2 \pi\left|V_{0}\right|^{2} \sin 2 \delta_{k}}{\left(1+i \Omega \tau_{p, k}\right)^{2}} .
$$

From above equation, it is found that $I_{y}^{\mathrm{I}}=0$ when the superconducting order parameter becomes zero above the transition temperature, and $I_{y}^{\mathrm{I}}$ changes sign when the chiral character $\eta \rightarrow-\eta$, in agreement with the experiment. 18 Moreover, from Eq. (28), one also finds that $I_{y}^{\mathrm{I}} \propto z_{i}^{3}$ (skew scattering), $I_{y}^{\mathrm{I}} \propto E_{0}$ (linear response), $I_{y}^{\mathrm{I}} \propto \Delta_{0}$ and $I_{y}^{\mathrm{I}} \propto \varepsilon_{k_{F}}$. These dependences of Channel I from our microscopic kinetic description confirm the results of the Kubo diagrammatic formalism of the skew scattering ${ }^{25,26}$ Nevertheless, it is noted that the frequency dependence at high frequency in our work $\left(I_{y}^{\mathrm{I}} \propto \Omega^{-2}\right)$ is different from the Kubo diagrammatic formalism of the skew scattering $\left(I_{y}^{\text {Kubo }} \propto \Omega^{-3}\right){ }^{25,26}$ This frequency-dependent difference comes from the different treatments of the impurity scattering between our kinetic description and Kubo diagrammatic formalism, whose detailed discussion is addressed in Sec. V

As for channel II, from Eq. (27), one finds that $I_{y}^{\mathrm{II}}$ is proportional to the quasiparticle correlation $\left(I_{y}^{\mathrm{II}} \propto \Gamma_{\mathbf{k} \pm}^{o}\right)$. Therefore, from Eq. (21), the excited quasiparticle correlation from the nonlinear effect is given by

$$
\rho_{\mathbf{k} \pm}^{q}=\frac{i\left(e E_{0}\right)^{2}}{i \Omega+\tau_{p, k}^{-1}} \frac{2 u_{k}^{3} v_{k}^{3}}{m E_{k}\left(\Omega \pm E_{\mathbf{k}}\right)}\left(1-6 \frac{\xi_{k} \varepsilon_{k}}{E_{k}^{2}} \cos ^{2} \theta_{\mathbf{k}}\right) .
$$

Consequently, from Eqs. (27) and (24), the anomalous Hall current for Channel II is given by

$$
I_{y}^{\mathrm{II}}=i \eta \nu^{2}\left(e E_{0}\right)^{3} \frac{e}{m} \int \frac{d \varepsilon_{k}}{8 m} \frac{3 \Delta_{0}^{6} \varepsilon_{k}^{2} \xi_{k}}{\left|\xi_{k}\right| E_{k}^{8} \Omega\left(\Omega^{2}-E_{k}^{2}\right)} \frac{n_{i} \Gamma_{k}^{\mathrm{II}} \tau_{p, k}^{2}}{\left(1+i \Omega \tau_{p, k}\right)^{2}},
$$

with $\Gamma_{k}^{\mathrm{II}}=\int d \delta \theta_{\mathbf{k k}^{\prime}}\left|V_{\mathbf{k k}^{\prime}}\right|^{2} \sin \delta \theta_{\mathbf{k k}^{\prime}} \sin 2 \delta \theta_{\mathbf{k k}^{\prime}}$. From above equation, one finds that $I_{y}^{\mathrm{II}}=0$ when the superconducting order parameter becomes zero above the transition temperature, and $I_{y}^{\mathrm{II}}$ changes sign when the chiral character $\eta \rightarrow-\eta$, similar to Channel I. Nevertheless, it is found that $I_{y}^{\mathrm{II}} \propto E_{0}^{3}$ (nonlinear response) and $I_{y}^{\mathrm{II}} \propto z_{i}^{2}$ (Born contribution), differing from Channel I.

This anomalous nonlinear channel from the Born contribution in the impurity scattering can be understood as follows. During the optical response, it is noted that the excited quasiparticle correlation from the nonlinear effect breaks the particle-hole symmetry, since Eq. (29) is not invariant under the particle-hole transformation $\xi_{k} \rightarrow-\xi_{k}{ }^{27}$ Moreover, it is revealed in the previous works $^{25,26}$ that in the chiral $p$-wave superconductor, the particle-hole asymmetry from the energy bandstructure can lead to the non-zero AHC from the Born contribution in the impurity scattering. Similarly, the broken particle-hole symmetry through the excited quasiparticle correlation here can also make the contribution to the AHC induction. Nevertheless, this exotic Channel II has long been overlooked in the literature due to the difficulty in treating the quasiparticle correlation in the previous semiclassical approach ${ }^{40}$ or including the nonlinear effect in Kubo diagrammatic formalism $\stackrel{25-27}{2}$

\section{NUMERICAL RESULTS}

In this section, we perform the full numerical calculation by solving the optical Bloch equations [Eqs. (5) and (8)] in the presence of the $\mathrm{HF}$ and impurity scattering $\left[\left.\partial_{t} \rho_{\mathbf{k}}\right|_{\text {scat }}=\left.U_{\mathbf{k}} \partial_{t} \rho_{\mathbf{k}}^{q}\right|_{\text {scat }} U_{\mathbf{k}}^{\dagger}\right.$ with Eq. (17) $]$ terms to investigate the $\mathrm{AHC}$ induction from impurity scattering in the chiral $p$-wave superconducting states.

The specific calculation is carried out in GaAs QW in which the influence of the spin-orbit coupling (SOC) is marginal and hence can be neglected ${ }^{75}$ The chiral $p$ wave superconductivity in QW can be realized through the proximity to superconducting $\mathrm{Sr}_{2} \mathrm{RuO}_{4}$. For the optical field, we choose a $\mathrm{THz}$ linear-polarized optical pulse, whose propagation direction is assumed to be perpendicular to the QW, i.e., the $\mathbf{e}_{\mathbf{z}}$ direction. The direction of the ac electric field is taken to be along $\mathbf{e}_{\mathbf{x}}$ without loss of generality. By considering the homogeneous limit of the optical field, from Eq. (6), one has

$$
\begin{aligned}
\mathbf{p}_{\mathbf{s}} & =(e / \Omega) E_{0} \mathbf{e}_{\mathbf{x}} \sin (\Omega t) \exp \left[-t^{2} /\left(2 \sigma_{t}^{2}\right)\right], \\
\partial_{t} \mathbf{p}_{\mathbf{s}} & =e E_{0} \mathbf{e}_{\mathbf{x}} \cos (\Omega t) \exp \left[-t^{2} /\left(2 \sigma_{t}^{2}\right)\right],
\end{aligned}
$$

with $\Omega$ and $\sigma_{t}$ being the frequency and width of the optical pulse. The parameters used in our computation are listed in Table [. In our investigation, the excited normal quasiparticle current always lies in the linear-response (i.e., small- $E_{0}$ ) regime (refer to Appendix C).

TABLE I: Parameters used in our calculation for GaAs QW in proximity to a chiral $p$-wave superconductor. $m_{0}$ stands for the free electron mass. $T$ is the temperature. $\sigma_{0}=e^{2} / \hbar$.

\begin{tabular}{llll}
\hline \hline$m / m_{0}$ & $0.067^{a}$ & $\kappa_{0}$ & $12.9^{a}$ \\
$n\left(\mathrm{~cm}^{-2}\right)$ & $2 \times 10^{11}$ & $\sigma_{t}(\mathrm{ps})$ & 4 \\
$T(\mathrm{mK})$ & 1 & $\Delta_{0}(\mathrm{meV})$ & $0.206^{b}$ \\
$E_{s}(\mathrm{kV} / \mathrm{cm})$ & 0.1 & $I_{0}$ & $10^{-4} E_{s} \sigma_{0}$ \\
$q\left(\mathrm{~nm}^{-1}\right)$ & $2 \pi / 60^{c}$ & $\Omega / \Delta_{0}$ & 8 \\
\hline \hline \multicolumn{2}{c}{${ }^{a}$ Ref. $75 .{ }^{b}$ Ref. $\left[{ }^{c}{ }^{c}\right.$ Ref. 79.}
\end{tabular}

We first focus on the case without the magnetic field. In this situation, with the translational symmetry, the spatial gradient terms in Eq. (5) can be neglected. We show that the conventional linear channel (Channel I) only dominates in the strong impurity interaction, while in the weak one, the anomalous nonlinear channel (Channel II) makes the dominant contribution.

Furthermore, we also study the AHC induction in the chiral $p$-wave superconducting state with a transverse conical magnetization $\mathbf{h}_{\mathbf{r}}=$ $h_{0}(\sin \theta \sin q x, \sin \theta \cos q x, \cos \theta) \stackrel{76-80}{.}$ Here, $\theta$ and $q$ are the conical angle and modulation wave vector, respectively. This transverse conical magnetization can be ap- 
plied through the proximity effect to the conical ferro-

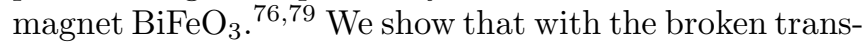
lational symmetry and hence the Galilean invariance by the magnetization, the intrinsic AHC, attributed from the anomalous velocity, is no longer zero. In this situation, comparison between the intrinsic AHC and extrinsic one from impurity scattering is presented.

Finally, the optical responses of the chiral $p$-wave superconducting order parameter including its superconducting phase and magnitude as well as the triplet-vector orientation are addressed.

\section{A. AHC in strong impurity interaction}

We first investigate the AHC induction in the relatively strong $\left(z_{i}=1\right)$ impurity interaction in the absence of the magnetic field. In this situation, the temporal evolutions of the induced anomalous Hall currents are plotted in Fig. 2 As seen from the figure, finite Hall currents $I_{y}$ with the oscillation frequency at the optical frequency $\Omega$ are observed even around zero temperature, and $I_{y}$ changes sign when the chiral character $\eta \rightarrow-\eta$ (yellow dotted curve), in agreement with the experiment $\underline{18}$ The excited quasiparticle current arises from the induced CM momentum of Cooper pairs through the acceleration driven by the ac electric field, since by removing the Berry curvature term in our full numerical calculation, the AHC in our work vanishes (green chain line). This quasiparticle-current excitation by the acceleration of the CM momentum, overlooked in the previous semiclassical approach,$\underline{40}$ confirms our analytic analysis in Sec. IIIB

Additionally, in the relatively strong impurity interaction, as shown in Fig. 2 the conventional linear channel (Channel I), i.e., skew scattering, shown by blue dashed curve which coincides with the full result (red solid curve), makes the dominant contribution in the $\mathrm{AHC}$ induction. In this situation, at small impurity density $\left(n_{i}<0.001 n\right)$, as shown in the inset (a) of Fig. 2, the induced anomalous Hall current (red solid curve) is linearly enhanced by impurity density. When $n_{i}>0.001 n$, the induced anomalous Hall current (red solid curve) is smaller than the fitted result from the $n_{i^{-}}$ linear relation (purple chain curve). This is due to the fact that at the large impurity density, the enhanced momentum scattering $\left[\tau_{p}^{-1}\right.$ in Eq. (28)] leads to the suppression of $I_{y}^{m}$. These impurity-density dependences confirm our analytic analysis [Eq. (28)] and Kubo diagrammatic formalism ${ }^{25,26}$ of the skew scattering. Particularly, from our analytic analysis [Eq. (28)], the AHC is obtained as $\sigma_{x y}^{I} \approx 0.308 \sigma_{0}$ when $n_{i}=0.002 n$, close to the value $\left(\sigma_{x y}^{t=t_{i}}=0.3 \sigma_{0}\right)$ fitted from the full numerical results at $t=-10.13$ ps [inset (b) in Fig. 2]. Nevertheless, $\sigma_{x y}^{I}$ is larger than the value $\sigma_{x y}^{m} \approx 0.11 \sigma_{0}$ fitted from the full numerical result of the Hall-current maxima [inset (c) in Fig. 2. This difference is due to the suppressed order parameter during the optical response, i.e., the excitation of the Higgs mode ${ }^{98-102}$ (refer to Sec. IVF), which

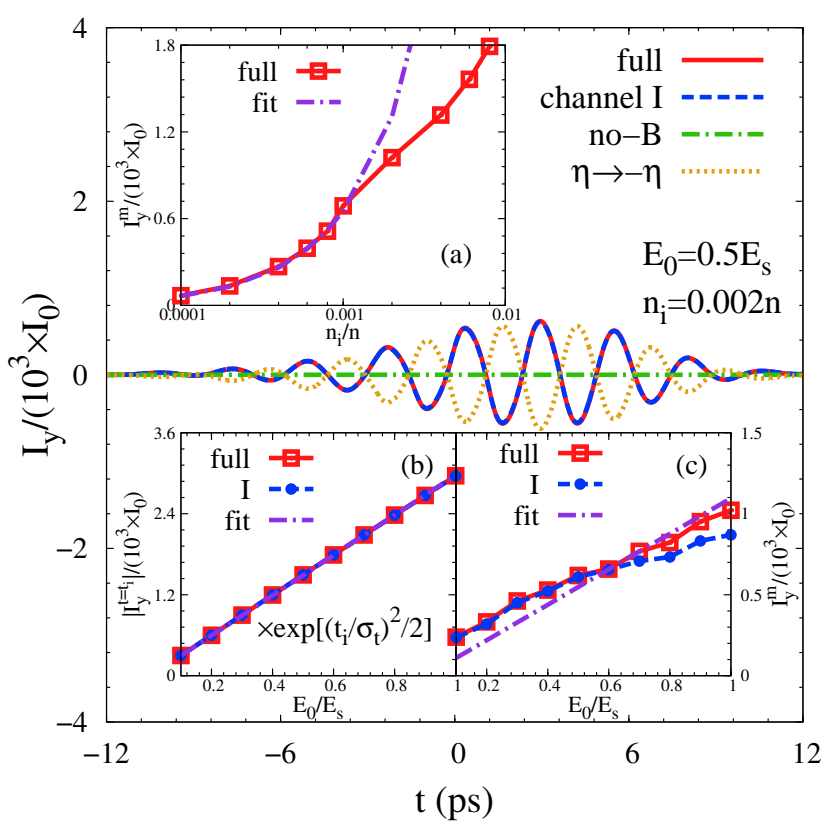

FIG. 2: (Color online) Induced anomalous Hall current $I_{y}$ in the relatively strong impurity interaction $\left(z_{i}=1\right)$. Green chain line: result without the Berry curvature; Blue dashed curve: result with only Channel I included by removing $Y_{\mathbf{k k}^{\prime}}$ terms and off-diagonal terms of $W_{\mathbf{k k}^{\prime}}^{a}$ in Eq. (17); Yellow dotted curve: result with the opposite chiral character $\eta \rightarrow-\eta$. The inset (a) shows the impurity-density dependence of the Hall-current maxima. Purple chain curve in inset (a): fitted result by using $I_{y} \propto n_{i}$. The inset (b) [(c)] shows the electric-field-strength dependence of the Hall current at $t_{i}=-10.13 \mathrm{ps}$ (at the maxima). Purple chain curves in insets (b) and (c): fitted results by using $I_{y} \propto E_{0}$.

suppresses the AHC according to our analytic analysis [Eq. (28)].

\section{B. AHC in weak impurity interaction}

We next investigate the AHC induction in the weak impurity interaction $\left(z_{i}=0.001\right)$. The induced anomalous Hall currents in this case are plotted in Fig. 3 As seen from the figure, finite Hall currents with the oscillation frequency $\Omega$ are observed around zero temperature due to the acceleration of the $\mathrm{CM}$ momentum of the Cooper pairs, and $I_{y}$ changes sign when the chiral character $\eta \rightarrow-\eta$ (yellow dotted curve), similar to the case in the strong impurity interaction.

Nevertheless, as shown in Fig. 3 and inset (a) of that figure, we find that Channel II (blue dashed curve) from the Born contribution, which has long been overlooked in the literature, makes the dominant contribution in the weak impurity interaction. Whereas the conventional linear channel (Channel I) from the skew scattering, shown by green chain curve, is marginal. This can be under- 


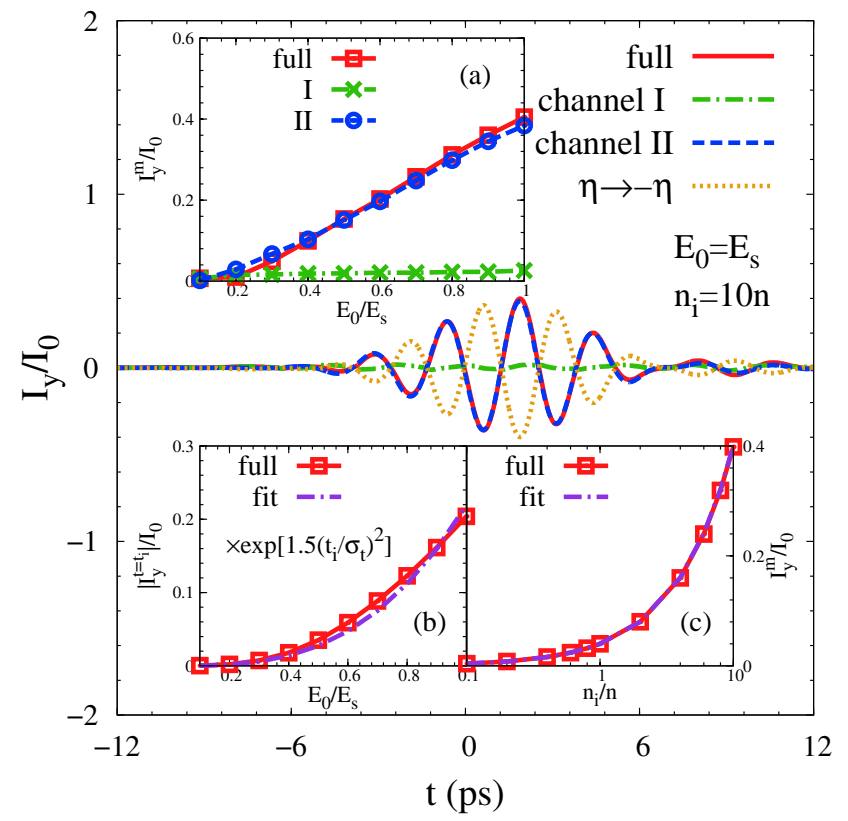

FIG. 3: (Color online) Induced anomalous Hall current $I_{y}$ in the weak impurity interaction $\left(z_{i}=0.001\right)$. Blue dashed (Green chain) curve: result with only Channel II (I) included by removing diagonal terms of $W_{\mathbf{k k}^{\prime}}^{a}\left(Y_{\mathbf{k k}^{\prime}}\right.$ terms and offdiagonal terms of $\left.W_{\mathbf{k k}^{\prime}}^{a}\right)$ in Eq. (17); Yellow dotted curve: result with the opposite chiral character $\eta \rightarrow-\eta$. The inset (a) $[(b)]$ shows the electric-field-strength dependence of the Hall current at the maxima (at $t_{i}=-4.4 \mathrm{ps)}$. The inset (c) shows the impurity-density dependence of the Hall-current maxima. Purple chain curve in inset (b) [(c)]: fitted result by using $I_{y} \propto E_{0}^{3}\left(I_{y} \propto n_{i}\right)$.

stood from the fact that the skew scattering $\left(\propto z_{i}^{3}\right)$ becomes marginal in the weak impurity interaction.

From the insets (b) and (c) of Fig. 3 it is seen that the induced Hall current $I_{y} \propto\left(e E_{0}\right)^{3}$ at $t_{i}=-4.4 \mathrm{ps}$ and the maxima $I_{y}^{m} \propto n_{i}$, in agreement with our analytic analysis of Channel II [Eq. (30)]. Nevertheless, from our analytic analysis [Eq. (30)], one has $I_{y}^{\mathrm{II}} \approx$ $7.5 \times 10^{-6} \sigma_{0}\left(E / E_{s}\right)^{2}$, smaller than the full numerical results $\left[I_{y} \approx 2.2 \times 10^{-5} \sigma_{0}\left(E / E_{s}\right)^{2}\right]$ in inset (b). The difference between the analytic and numerical results comes from the neglected scattering term $Y_{\mathbf{k k}^{\prime}}$ in Sec. IIIC. which also contributes to Channel II. Additionally, although the Hall-current maximum $I_{y}^{m}$ [inset (a) of Fig. 3] increases nonlinearly with the electric-field strength, $I_{y}^{m}$ deviates from the analytic analysis $\left[I_{y} \propto\left(e E_{0}\right)^{3}\right]$ due to the complex nonlinear excitation and scattering process in the temporal evolution.

\section{Impurity interaction strength dependence of AHC}

In this section, we address the impurity interaction strength dependences of the induced AHC, which are plotted in Fig. 4. As seen from the figure, Channel II (I) [green dotted curve (blue dashed curve)] makes the dominant contribution in the weak (strong) impurity interaction when $z_{i}<0.02\left(z_{i}>0.02\right)$. This is because that Channel I (II) arises from the skew scattering 25,26 (Born contribution in the impurity scattering), and hence, based on the Kubo formalism, 25.26 the induced $\mathrm{AHC}$ is proportional to $z_{i}^{3}\left(z_{i}^{2}\right)$, in agreement with our numerical result shown in the inset (a) [(b)] of Fig. 团and analytic one in Eq. (28) [Eq. (30)]. It is noted that in the insets (a) and (b), the smaller numerical results than the analytic ones when $z_{i}>0.04$ is due to the suppression from the enhanced momentum relaxation.

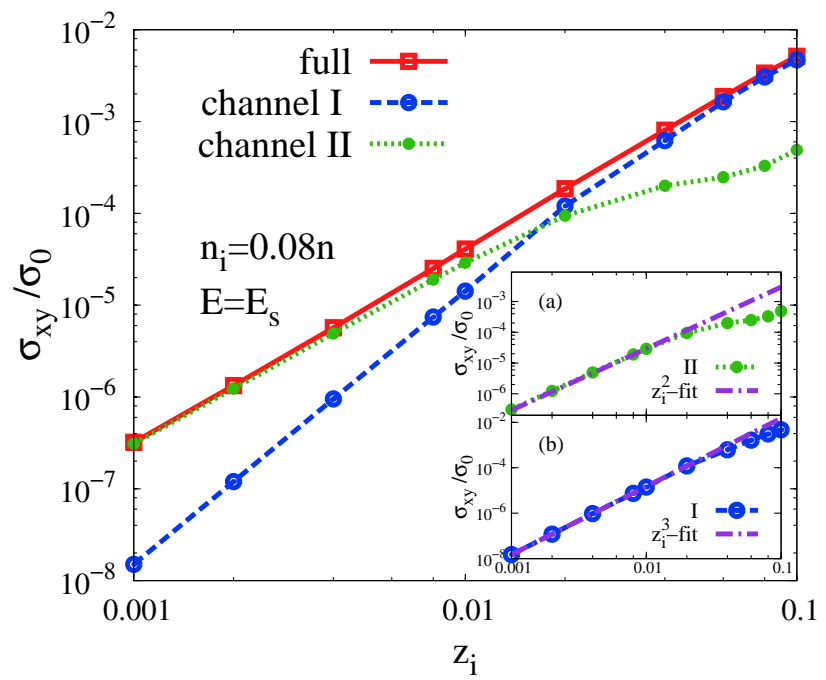

FIG. 4: (Color online) AHC (fitted from the Hall-current maxima) versus impurity interaction strength. Green dotted (Blue dashed) curve: result with only Channel II (I) included by removing diagonal terms of $W_{\mathbf{k k}^{\prime}}^{a}\left(Y_{\mathbf{k k}^{\prime}}\right.$ terms and off-diagonal terms of $\left.W_{\mathbf{k k}^{\prime}}^{a}\right)$ in Eq. (17). The inset (a) [(b)] shows the fitted results by using $\sigma_{x y} \propto z_{i}^{2}\left(\sigma_{x y} \propto z_{i}^{3}\right)$.

\section{Intrinsic channel by introducing transverse conical magnetization}

Finally, we show that in the presence of the transverse conical magnetization, the intrinsic $\mathrm{AHC}$ is no longer zero. Specifically, with the transverse conical magnetization, the translational symmetry is broken. In this situation, considering the fact that the magnetization varies smoothly with the length scale $l=2 \pi / q \gg \xi$ ( $\xi$ denotes the superconducting coherence length), we 
treat the spatial derivative of $\mathbf{h}_{\mathbf{R}}$ perturbatively. This treatment can be simplified $103-105$ by transforming the optical Bloch equations [Eq. (5)] into the helical space (in which the spin axis is parallel to $\mathbf{h}_{\mathbf{R}}$ ) through a unitary transformation matrix $Q_{\mathbf{R}}=e^{-i\left(\boldsymbol{\alpha}_{\mathbf{R}} \cdot \tilde{\boldsymbol{\sigma}}\right) \tau_{3}}$ via keeping non-Abelian gauge invariance ${ }^{84}$ (more details of the derivation refer to Appendix A). Here, $\alpha_{\mathbf{R}}=$ $\frac{\pi}{2}(\sin (\theta / 2) \sin (q x), \sin (\theta / 2) \cos (q x), \cos (\theta / 2))$. In the helical space, the magnetization becomes homogeneous. In this situation, following the previous works $\stackrel{103-105}{=}$ one can neglect the spatial derivatives and the optical Bloch equations read:

$$
\begin{aligned}
& \partial_{T} \rho_{\mathbf{k}}^{h}+i\left[\left(\begin{array}{cc}
\xi_{k}+\mu_{\mathrm{eff}}+h_{0} \sigma_{z} & \left(\mathbf{d}^{h} \cdot \boldsymbol{\sigma}\right) e^{i \eta \theta_{\mathbf{k}}} \\
\left(\mathbf{d}^{h} \cdot \boldsymbol{\sigma}\right) e^{-i \eta \theta_{\mathbf{k}}} & -\xi_{k}-\mu_{\mathrm{eff}}-h_{0} \sigma_{z}
\end{array}\right), \rho_{\mathbf{k}}^{h}\right] \\
& +\frac{1}{2}\left\{\partial_{t} \mathbf{p}_{\mathbf{s}} \tau_{3}, \partial_{\mathbf{k}} \rho_{\mathbf{k}}^{h}\right\}+\frac{1}{2}\left\{\left[\left(\mathbf{A}_{\mathbf{s}} \cdot \tilde{\boldsymbol{\sigma}}\right) \mathbf{e}_{\mathbf{x}}, h_{0} \tilde{\sigma}_{z}\right], \partial_{\mathbf{k}} \rho_{\mathbf{k}}^{h}\right\} \\
& +i\left[\frac{\mathbf{p}_{\mathbf{s}}^{2}}{2 m} \tau_{3}, \rho_{\mathbf{k}}\right]-\frac{i}{2}\left\{\frac{k_{x}}{m} \tau_{3},\left[\left(\mathbf{A}_{\mathbf{s}} \cdot \tilde{\boldsymbol{\sigma}}\right) \tau_{3}, \rho_{\mathbf{k}}^{h}\right]\right\} \\
& +\frac{i}{2}\left[\frac{\mathbf{p}_{\mathbf{s}} \cdot \mathbf{e}_{\mathbf{x}}}{m} \tau_{3},\left\{\left(\mathbf{A}_{\mathbf{s}} \cdot \tilde{\boldsymbol{\sigma}}\right), \rho_{\mathbf{k}}^{h}\right\}\right]=\left.\partial_{t} \rho_{\mathbf{k}}\right|_{\text {scat }},
\end{aligned}
$$

where $\mathbf{A}_{\mathbf{s}}=\partial_{x} \boldsymbol{\alpha}_{\mathbf{R}}=\frac{q \pi}{2} \sin \left(\frac{\theta}{2}\right)(\cos (q x),-\sin (q x), 0)$; $\rho_{\mathbf{k}}^{h}=Q_{\mathbf{R}}^{\dagger} \rho_{\mathbf{k}} Q_{\mathbf{R}}$ and $\left(\mathbf{d}^{h} \cdot \boldsymbol{\sigma}\right)=e^{i\left(\boldsymbol{\alpha}_{\mathbf{R}} \cdot \boldsymbol{\sigma}\right)}(\mathbf{d} \cdot \boldsymbol{\sigma}) e^{-i\left(\boldsymbol{\alpha}_{\mathbf{R}} \cdot \boldsymbol{\sigma}\right)}$ are the density matrix and effective triplet vector in the helical space, respectively.

In Eq. (33), the fourth term corresponds to the drive field from the SG force. The sixth term acts as an effective SOC from the spin-rotational transformation ${ }^{84,103}-105$ The seventh term, which is the coupling between the optical field and magnetization, acts as an excited effective in-plane magnetic field along $\mathbf{A}_{s}$. In this situation, with $\mathbf{p}_{\mathbf{s}}$ and $\partial_{t} \mathbf{p}_{\mathbf{s}}$ given in Eqs. (31) and (32), we perform the numerical calculation by solving the optical Bloch equations [Eqs. (33) and (8)] in the presence of the impurity scattering. We find that the induced effective chemical potential and the induced current have no spatial dependence (not shown). This indicates the homogeneous condition $\left(\nabla_{\mathbf{R}} \mu_{\mathrm{eff}}=0\right)$ to use Eqs. (31) and (32) is still satisfied here.

We first focus on the clean limit. In this situation, finite anomalous Hall currents with the oscillation frequency at the optical frequency $\Omega$, plotted in Fig. 5, are observed. Particularly, the anomalous Hall current comes from the linear response, since $I_{y}^{m}$ is linearly proportional to $E_{0}$, as shown in the inset of Fig. 5 .

The induced anomalous Hall current here is attributed to the broken translational symmetry and hence the Galilean invariance by the transverse conical magnetization. This can also be understood as follows. Through the SOC, i.e., momentum-dependent effective magnetic field, the spin-polarized quasiparticles are induced with a net quasiparticle momentum driven by the ac electric field. Consequently, the SG force drives the spinpolarized quasiparticles to form the net anomalous current via the anomalous velocity (i.e., Berry curvature). This analysis is demonstrated in Fig. 5, since the result with only the drive effect, SG force and SOC included

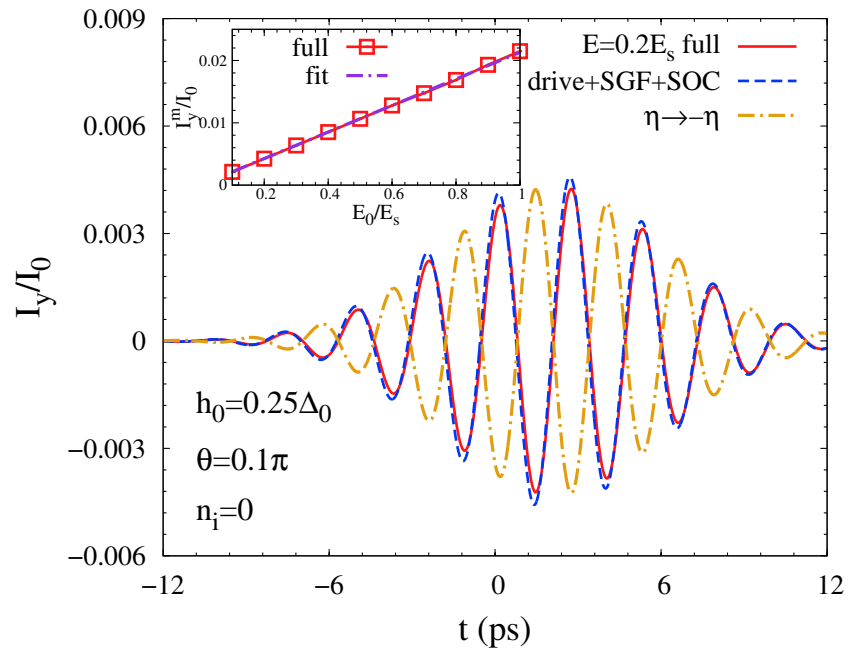

FIG. 5: (Color online) Induced anomalous Hall current $I_{y}$ in the presence of the transverse conical magnetization in the clean limit. Blue dashed curve: result with only the drive effect, SOC and SG force included by setting $\mathbf{p}_{s}=0$. Yellow chain curve: result with the opposite chiral character $\eta \rightarrow-\eta$. The inset shows electric-field-strength dependence of the Hallcurrent maxima. Purple chain curve in the inset: fitted result by using $I_{y} \propto E_{0}$.

(blue dashed curve) almost coincides with the full one (red solid curve).

Moreover, based on the analysis above, we give the analytical formula for the anomalous Hall current induced by the transverse conical magnetization in the linear response (refer to Appendix D), which is approximately given by:

$$
I_{y}^{t}=16 E_{0} \sigma_{0} \pi^{3} \eta \sin ^{4}\left(\frac{\theta}{2}\right) \frac{h_{0}^{4} \epsilon_{q}^{2} \Delta_{0}^{2}}{\Omega^{8}} \int d\left(\frac{\epsilon_{k}}{\Delta_{0}}\right)\left(\frac{\Delta_{0}}{E_{k}}\right)^{3} .
$$

From above equation, it is found that $I_{y}^{t}$ changes sign when the chiral character $\eta \rightarrow-\eta$, in consistence with the full numerical result (brown chain curve) in Fig. 5 , and $I_{y}^{t}=0$ when the superconducting order parameter becomes zero above the transition temperature. This indicates that the induced intrinsic anomalous Hall effect is exactly related to the chiral $p$-wave superconductivity, similar to the extrinsic AHC induction from impurity scattering. Furthermore, by restoring the translational symmetry via setting conical angle $\theta=0$ or modulation wave vector $q=0$, one has $I_{y}^{t}=0$ from Eq. (34), in consistence with the Galilean invariance. Additionally, from Eq. (34), the AHC is obtained as $\sigma_{x y}^{t} \approx 2.02 \times 10^{-6} \sigma_{0}$, close to the value $\left(\sigma_{x y}^{\mathrm{fit}}=2.1 \times 10^{-6} \sigma_{0}\right)$ fitted from the full numerical results (inset in Fig. [5). 


\section{E. Comparison between extrinsic and intrinsic channels}

In this part, we compare the extrinsic AHC induction channel from the impurity scattering with the intrinsic one from transverse conical magnetization. As shown in Fig. 6. the intrinsic channel from magnetization (blue dashed curve), which is nearly invariant with the increase of the impurity density, dominates at small impurity density. The extrinsic induction channel from the impurity scattering (green dotted curve), is enhanced by the increase of impurity density and makes the dominant contribution at large impurity density.

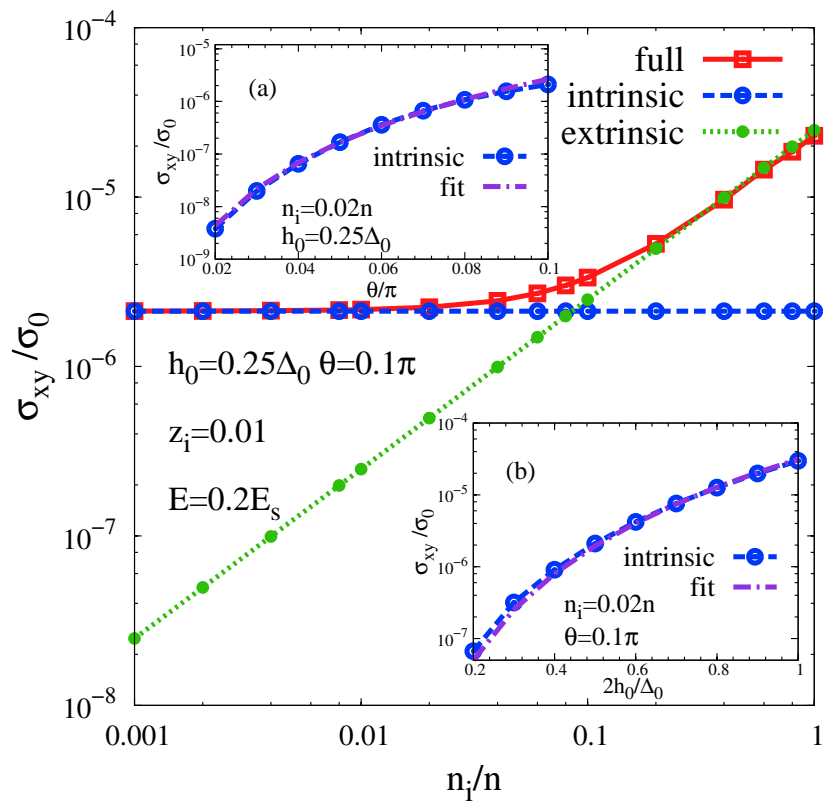

FIG. 6: (Color online) AHC (fitted from the Hall-current maxima) versus impurity density in the presence of the transverse conical magnetization and impurity scattering. Blue dashed (Green dotted) curve: result with only the intrinsic (extrinsic) AHC included by removing $W_{\mathbf{k k}^{\prime}}^{a}$ and $Y_{\mathbf{k} \mathbf{k}^{\prime}}$ terms in Eq. (17) [SG force in Eq. (33)]. The inset (a) [(b)] shows conical angle $\theta$ (magnetization strength $h_{0}$ ) dependence of the intrinsic AHC. Purple chain curve in the inset (a) [(b)]: fitted result by using $I_{y} \propto \sin ^{4}(\theta / 2)\left(I_{y} \propto h_{0}^{4}\right)$.

Moreover, the intrinsic channel from the transverse conical magnetization can be enhanced though the enhancement of the translational symmetry breaking by increasing the strength $h_{0}$ and conical angle $\theta$, as shown in the insets (a) and (b) of Fig. 6] respectively, in good agreement with the analytic analysis [Eq. (34)]. This provides a scheme to experimentally distinguish these two channels.

\section{F. Optical response of the superconducting order parameter}

Finally, we study the optical response of the superconducting order parameter. We first investigate the effective chemical potential $\mu_{\text {eff }}$ in Fig. 77(a), which is related to the phase of the superconducting order parameter (known as the collective mode $\mathrm{e}^{30-38,41}$ ). Compared to the situation for the $s$-wave superconducting state in the previous work ${ }^{41}$ where the pump and drive effects both play important roles, for the chiral $p$-wave case, the drive effect (blue dashed curve) dominates the excited oscillation of $\mu_{\text {eff }}$ with frequency $2 \Omega$ (red solid curve). This difference lies in the weak impurity scattering in our work, considering the fact that the impurity scattering in the chiral $p$-wave superconducting state is essentially weak in order to achieve the superconducting phase. . $, 6,12,13^{-1}$ Consequently, the suppression of the drive effect from impurity scattering in the previous work ${ }^{41}$ for $s$-wave case is marginal here 106

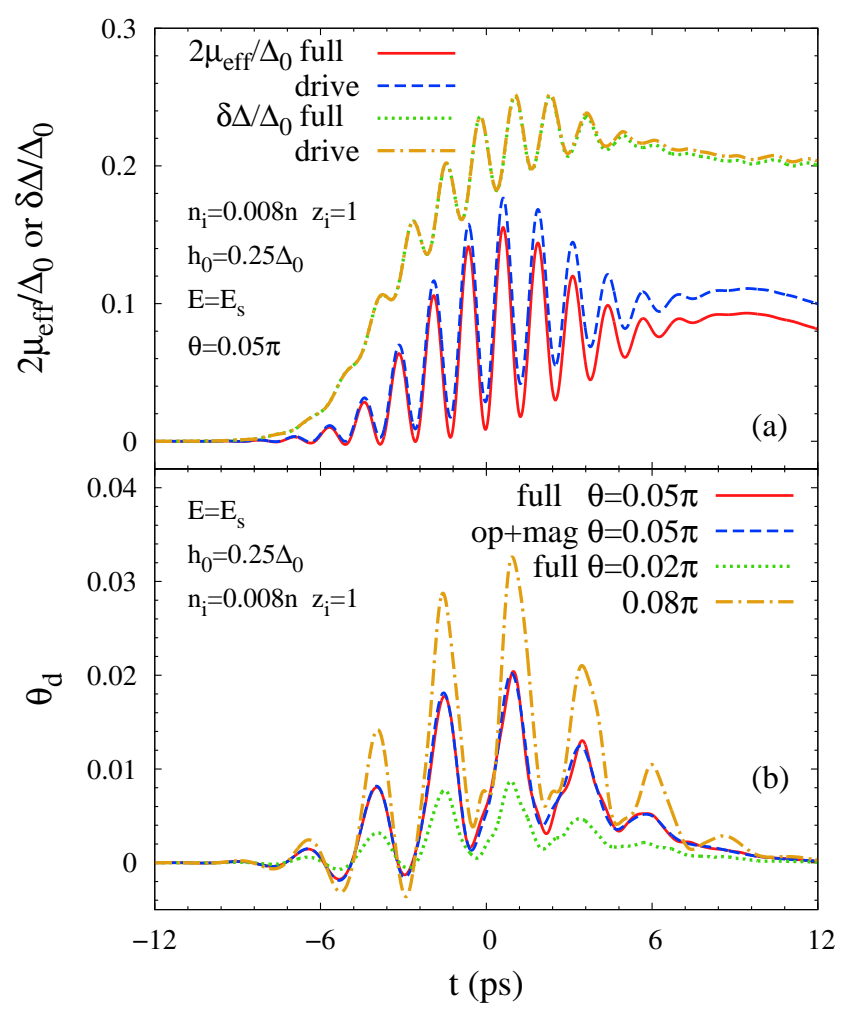

FIG. 7: (Color online) Temporal evolutions of the effective chemical potential, magnitude of the triplet vector $\delta \Delta=$ $\sum_{\mathbf{k}}\left(\left|\mathbf{d}_{\mathbf{0}}\right|-\left|\mathbf{d}_{\mathbf{k}}\right|\right) /\left(\sum_{\mathbf{k}} 1\right)$ in (a) and longitude angle of the triplet vector $\theta_{\mathbf{d}}=\sum_{\mathbf{k}} \theta_{\mathbf{d}_{\mathbf{k}}} /\left(\sum_{\mathbf{k}} 1\right)$ in (b). Blue dashed and yellow chain curves in (a): results with only the drive effect included. Blue dashed curve in (b): result without the SOC and SG force in Eq. (33).

During the optical response with the transverse conical magnetization, we also find that the triplet vector oscil- 
lates as $\mathbf{d}=d(t)\left(\cos (q x) \sin \theta_{\mathbf{d}}(t),-\sin (q x) \theta_{\mathbf{d}}(t), \cos \theta_{\mathbf{d}}(t)\right)$ with the excited space-independent oscillations in the magnitude $d(t)$ [Fig. [7(a)] and longitude angle $\theta_{d}(t)$ [Fig. 7(b)]. Specifically, as shown in Fig. 77(a), the oscillation of the magnitude (green dotted curve) with frequency $2 \Omega$ (referred to as Higgs mode $41,98-102$ ) is dominated by the drive effect (yellow chain curve), similar to the $s$-wave case. 41

As for the excited triplet-vector rotation (red solid curve) shown in Fig. 7(b), the excited effective magnetic field [seventh term in Eq. (33)], shown by blue dashed curve, makes the dominant contribution. This can be understood from the fact that the triplet vector in the static situation tends to be parallel to the magnetic field ${ }^{60,107-112}$ Consequently, in the optical response, the excited effective in-plane magnetic field $\left(\mathbf{h}_{\mathrm{in}}^{\text {eff }} \propto p_{s} \mathbf{A}_{s}\right)$, can induce the oscillating in-plane component of the triplet-vector along $\mathbf{A}_{s}=(\cos q x,-\sin q x, 0)$ with frequency $\Omega$. Particularly, this optically excited tripletvector rotation can be enhanced through the enhancement of the effective in-plane magnetic field by increasing the conical angle, as shown Fig. 7(b). This prediction provides a scheme to experimentally manipulate the triplet vector via the optics methods.

\section{SUMMARY AND DISCUSSION}

In summary, with the impurity scattering included, by extending the gauge-invariant optical Bloch equation from the $s$-wave superconducting state into the chiral $p$ wave state, we systematically investigate the AHC induction in the chiral $p$-wave superconducting states. In this study, we focus on $\mathrm{THz}$ optical pulse response in GaAs QW in proximity to the chiral $p$-wave superconductor $\mathrm{Sr}_{2} \mathrm{RuO}_{4}$. However, the predictions revealed in our study can still shed light on continuous-wave optical response in the chiral $p$-wave superconductor. Particularly, in contrast to the well established Kubo formalism, ${ }^{25-27}$ we provide a microscopic kinetic description for the AHC induction and reveal a new AHC induction channel from impurity scattering in our work.

Specifically, we first demonstrate that the intrinsic AHC without magnetic field is zero as a consequence of the Galilean invariance, in agreement with the previous works ${ }^{23-27}$ As for the extrinsic AHC, we show that even at zero temperature, there exists optically excited non-zero normal quasiparticle current, and a finite anomalous Hall current from the impurity scattering is obtained, in consistence with the experiment. 18 Particularly, the excited normal quasiparticle current arises from the induced CM momentum of Cooper pairs through the acceleration driven by the ac electric field, which has been overlooked in the previous semiclassical approach. 40 Specifically, as pointed out in the previous works, $\stackrel{41-50}{\simeq}$ the induction of a large CM momentum excites Bogoliubov quasiparticles by breaking the Cooper pairs, i.e., forming the blocking region with the markedly suppressed anomalous correlation, leading to the induced quasiparticle current even at zero temperature. We find that there exist two AHC induction channels from the impurity scattering: Channel I, conventional linear channel, in which the $\mathrm{AHC}$ is induced from the skew scattering in the linear response; Channel II, anomalous nonlinear channel, where the AHC is induced from the Born contribution due to the broken particle-hole symmetry by the nonlinearly excited quasiparticle correlation. Both induction channels change sign under the opposite chiral characters and vanishes when the superconducting order parameter approaches zero above the transition temperature, in consistence with the experiment 18

Particularly, we point out that Channel I from our microscopic kinetic description mostly confirms the results of Kubo diagrammatic formalism of the skew scattering including the electron-density, impurity-density and order-parameter dependences. Nevertheless, the frequency dependence of $\mathrm{AHC}$ at high frequency in our work $\left(\sigma_{x y}^{\mathrm{I}} \propto \Omega^{-2}\right)$ is different from the Kubo diagrammatic formalism of the skew scattering $\left(\sigma_{x y}^{\text {Kubo }} \propto \Omega^{-3}\right)$. This frequency-dependent difference comes from the different scattering treatments. Specifically, for Kubo formalism, the skew scattering is treated within perturbation theory by picking up particular crossed Feynman diagrams..$^{25-27}$ In our work, we use the scattering $T$ matrix method, which has been widely applied to the kinetic description in the literature ${ }^{40,92-97}$ Following the standard treatment of energy $E$ in the $T$-matrix $T_{\mathbf{k k}^{\prime}}(E)$ in normal metals $\left(E=E_{F}\right)$, we takes $E=E_{k}$ here, exactly same as the previous work for superconducting state .40 In the normal state, these two approaches for the AHC mostly give inconsistent results with each other and are considered as complementary techniques, ${ }^{95}$ differing from the longitudinal conductivity in which the Kubo formalism via picking up the self-consistent ladder Feynman diagrams agrees well with the kinetic description. For the chiral $p$-wave superconducting state, the prediction of the AHC from our microscopic kinetic description agrees qualitatively with the present experiment, 18 similar to the well established Kubo formalism. ${ }^{25-27}$ Further experiments on the frequency dependence would help to determine which method better describes the AHC in the chiral $p$-wave superconducting state. Nevertheless, to elucidate a complete link between the kinetic description and Kubo formalism, a gauge-invariant extension for the Kubo formalism should be developed, since differing from our gauge-invariant description, a special gauge with zero scalar potential, zero superconducting phase and finite vector potential is chosen in the Kubo approach in the literature ${ }^{25-27}$ Nevertheless, from the gauge structure re-

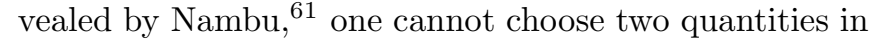
the vector potential, scalar potential, and superconducting phase to be zero.

As for Channel II in our work, to the best of our knowledge, this exotic channel has long been overlooked in the literature, due to the difficulty in treating the quasiparticle correlation in the previous semiclassical approach ${ }^{40}$ 
or including the nonlinear effect in Kubo diagrammatic formalism ${ }^{25-27}$ Nevertheless, we find that the conventional linear channel (Channel I) only dominates in the strong impurity interaction, whereas in the weak impurity interaction, the anomalous nonlinear channel (Channel II) makes the dominant contribution. Based on this, Channel II may also play an important role in $\mathrm{Sr}_{2} \mathrm{RuO}_{4}$, considering the fact that the impurity scattering in the chiral $p$-wave superconducting state is essentially weak in order to achieve the superconducting phase,$\stackrel{3,6,12,13}{ }$ In addition, we point out that even in the relatively strong impurity interaction, Channel II can also play an important role in the pump-probe spectroscopy. Specifically, the pump pulse can markedly excite the quasiparticle correlation in the nonlinear response. Consequently, Channel II, which arises from the excited quasiparticle correlation, will make an important contribution in the probe of Kerr rotation.

Furthermore, motivated by the recent observed penetration of the superconductivity from $\mathrm{Sr}_{2} \mathrm{RuO}_{4}$ into the normal metal through a ferromagnet in metal/ferromagnet/ $\mathrm{Sr}_{2} \mathrm{RuO}_{4}$ junctions,$\underline{69}$ we also study the AHC induction in chiral $p$-wave superconducting QWs in the presence of a magnetization. In our work, we consider a specific transverse conical magnetization, $\underline{\underline{76}} \underline{\underline{80}}$ which breaks the translational symmetry and hence the Galilean invariance. In this situation, the intrinsic AHC, attributed from the anomalous velocity, is no longer zero. We show that this intrinsic AHC induction changes sign under the opposite chiral characters and vanishes when the superconducting order parameter approaches zero. This indicates that the induced intrinsic AHC is exactly related to the chiral $p$-wave superconductivity, similar to the extrinsic one from impurity scattering. Comparison between the intrinsic and extrinsic AHCs from impurity scattering is also addressed in our work. We show that the intrinsic AHC can be enhanced though the enhancement of the translational symmetry breaking by increasing the strength or conical angle of the transverse conical magnetization. Whereas the extrinsic AHC can be enhanced by increasing impurity density. These dependences provide a scheme to experimentally distinguish the intrinsic and extrinsic AHC induction channels.

Finally, the optical response of the chiral $p$-wave superconducting order parameter is addressed. We reveal that the drive effect of the particles dominates the optically excited effective chemical potential (related to the phase of the superconducting order parameter) in the chiral $p$-wave superconducting state, differing from the $s$-wave case where both the pump and drive effects play important roles. Moreover, it is also found that the optical field excites the rotation of the triplet vector in the presence of the transverse conical magnetization. This provides a scheme to experimentally manipulate the triplet vector via the optics methods.

\section{Acknowledgments}

This work was supported by the National Natural Science Foundation of China under Grants No. 11334014 and No. 61411136001.

\section{Appendix A: Gauge invariant optical Bloch equation}

In this section, we construct the optical Bloch equations for chiral $p$-wave superconducting state in the presence of the optical field and magnetization. We begin with the generalized Hamiltonian:

$H_{0}=\left[\xi_{-i \boldsymbol{\nabla}-e \mathbf{A}(x) \tau_{3}-\mathbf{A}_{s}^{i}(x) \tilde{s}^{i} \tau_{3}}+e \phi(x)+\psi_{s}^{i}(x) \tilde{s}^{i}\right] \tau_{3}+\hat{\Delta}_{\mathbf{k}}^{\mathbf{r}}\left(\mathbf{d}_{0}\right)$,

with $\tilde{s}^{i}=\tilde{\sigma}_{i} / 2$ being the spin vector. Here, $\psi_{s}^{i}$ denotes the magnetic field and $\mathbf{A}_{s}^{i}$ represents the effective SOC coefficient. Under a spin-rotational transformation $Q(x)=e^{i[\boldsymbol{\alpha}(x) \cdot \tilde{\mathbf{s}}] \tau_{3}}$, there exists a non-Abelian gauge ${ }^{84}$ structure:

$$
\begin{aligned}
A_{s \mu}^{i} \tilde{s}^{i} \tau_{3} & \rightarrow A_{s \mu}^{i} \tilde{s}^{i} \tau_{3}-\partial_{\mu} \alpha^{i} \tilde{s}^{i} \tau_{3}+i\left[\alpha^{i} \tilde{s}^{i}, A_{s \mu}^{k} \tilde{s}^{k}\right] \\
\mathbf{d} \cdot \boldsymbol{\sigma} & \rightarrow \mathbf{d} \cdot \boldsymbol{\sigma}+i[\boldsymbol{\alpha}(x) \cdot \mathbf{s}, \mathbf{d} \cdot \boldsymbol{\sigma}]
\end{aligned}
$$

where the four vectors are $A_{s \mu}^{i}=\left(\psi_{s}^{i}, \mathbf{A}_{\mathbf{s}}{ }^{i}\right), 84$ It is noted that this non-Abelian structure in Eqs. (A2) and (A3) is similar to the Abelian structure in Eqs. (3) and (4) for the optical field. Particularly, the presence of the triplet vector breaks the spin-rotational symmetry ${ }^{84}$ [Eq. [A3)], similar to the fact that the order parameter breaks the global U(1) symmetry [Eq. (4)]. Consequently, in the optical Bloch equations, besides the Abelian gauge invariance, the non-Abelian gauge invariance also has to be retained to elucidate the complete physical picture, as mentioned in the introduction.

Specifically, the optical Bloch equations can be constructed from the lesser Green function $G_{12}^{<}=i\left\langle\Psi_{2}^{\dagger} \Psi_{1}\right\rangle$, in which $1 / 2=x_{1} / x_{2}$ and \langle\rangle denotes the ensemble average $83,85,87$ With the spin-rotational transformation $\Psi(x) \rightarrow Q(x) \Psi(x)$, the Green function $G_{12}^{<} \rightarrow$ $Q\left(x_{1}\right) G_{12}^{<} Q^{\dagger}\left(x_{2}\right)$. Since in the kinetic equations in the quasiparticle approximation, only the $\mathrm{CM}$ coordinates are retained, 85 the gauge structure cannot be easily realized in the kinetic equations constructed from $G_{12}^{<}$. Nevertheless, as pointed out in Ref. 41, the gauge invariance can be retained by introducing the Wilson line ${ }^{82-86}$ to construct the gauge-invariant Green function, which is constructed as

$$
\begin{aligned}
G_{12}^{g<}= & \mathrm{P} e^{-i \int_{x_{1}}^{R} d x^{\mu} A_{\mu} \tau_{3}} e^{-i \int_{x_{1}}^{R} d x^{\nu} A_{s \nu}^{i} \tilde{s}^{i} \tau_{3}} \\
& \times G_{12}^{<} e^{-i \int_{R}^{x_{2}} d x^{\nu} A_{s \nu}^{i} \tilde{s}^{i} \tau_{3}} e^{-i \int_{R}^{x_{2}} d x^{\mu} A_{\mu} \tau_{3}} .
\end{aligned}
$$

In Eq. (A4), $d x^{\mu}=(d t,-d \mathbf{r})$ and $R=(\mathbf{R}, T)=\left(x_{1}+\right.$ $\left.x_{2}\right) / 2$ are the $\mathrm{CM}$ coordinates. "P" indicates that the line integral is path dependent. By the spin-rotational transformation $Q(x)=e^{i[\boldsymbol{\alpha}(x) \cdot \tilde{\mathbf{s}}] \tau_{3}}$, the gauge-invariant Green function is transformed as $G_{12}^{g<} \rightarrow Q(R) G_{12}^{g<} Q^{\dagger}(R)$, in 
which the transformation operator only depends on the CM coordinates.

Finally, by choosing the path to be the straight line

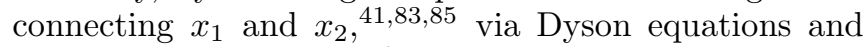
gradient expansion $83,85,87$ (the details of the derivation can be referred to Ref. 41), the optical Bloch equations are written as

$$
\begin{aligned}
& \partial_{T} \rho_{\mathbf{k}}+i\left[\left(\xi_{k}+e \phi+\phi_{s}^{i} \tilde{s}^{i}\right) \tau_{3}+H_{\mathbf{k}}^{\mathrm{HF}}+\hat{\Delta}_{\mathbf{k}}^{\mathbf{R}}\left(\mathbf{d}_{\mathbf{0}}\right), \rho_{\mathbf{k}}\right] \\
& -i\left[\frac{1}{8 m} \tau_{3}, \boldsymbol{\nabla}_{\mathbf{R}}^{2} \rho_{\mathbf{k}}\right]+\left\{\frac{\mathbf{k}}{m} \tau_{3}, \boldsymbol{\nabla}_{\mathbf{R}} \rho_{\mathbf{k}}\right\}+\frac{1}{2}\left\{e \mathbf{E} \tau_{3}, \partial_{\mathbf{k}} \rho_{\mathbf{k}}\right\} \\
& -\left[\frac{e \boldsymbol{\nabla}_{\mathbf{R}} \cdot \mathbf{A}}{4 m} \tau_{3}, \tau_{3} \rho_{\mathbf{k}}\right]-\left[\frac{e \mathbf{A}}{2 m} \tau_{3}, \tau_{3} \boldsymbol{\nabla}_{\mathbf{R}} \rho_{\mathbf{k}}\right] \\
& -\left[\frac{\tau_{3}}{8 m},\left[\left(\boldsymbol{\nabla}_{\mathbf{R}} \cdot \mathbf{A}_{s}^{i}\right) \tilde{s}^{i} \tau_{3}, \rho_{\mathbf{k}}\right]\right]-\left[\frac{\tau_{3}}{4 m},\left[\mathbf{A}_{s}^{i} \tilde{s}^{i} \tau_{3}, \nabla_{\mathbf{R}} \rho_{\mathbf{k}}\right]\right] \\
& +\frac{i}{2}\left[\frac{e \mathbf{A}}{m} \tau_{3},\left\{\mathbf{A}_{s}^{i} \tilde{s}^{i}, \rho_{\mathbf{k}}\right\}\right]-\frac{i}{2}\left[\frac{e \mathbf{k}}{m} \tau_{3},\left\{\mathbf{A}_{s}^{i} \tilde{s}^{i} \tau_{3}, \rho_{\mathbf{k}}\right\}\right] \\
& +\frac{1}{2}\left\{i\left[\mathbf{A}_{s}^{i} \tilde{s}^{i}, \phi_{s}^{j} \tilde{s}^{j}\right]-\nabla_{\mathbf{R}} \phi_{s}^{i} \tilde{s}^{i} \tau_{3}, \partial_{\mathbf{k}} \rho_{\mathbf{k}}\right\}+\left[\frac{i A^{2}}{2 m} \tau_{3}, \rho_{\mathbf{k}}\right] \\
& +i\left[\frac{1}{4 m} \tau_{3}, \frac{A_{s}^{2}}{4} \rho_{\mathbf{k}}+\mathbf{A}_{s}^{i} \tilde{s}^{i} \rho_{\mathbf{k}} \mathbf{A}_{s}^{i} \tilde{s}^{i}\right]=\left.\partial_{t} \rho_{\mathbf{k}}\right|_{\text {scat }} .
\end{aligned}
$$

Here, $\mathbf{E}=-\boldsymbol{\nabla}_{\mathbf{R}} \phi-\partial_{t} \mathbf{A}$. It is noted that with the Abelian [Eqs. (3) and (4)] and non-Abelian [Eqs. (A2) and (A3)] gauge structures, Eq. (A5 is gauge invariant for both the Abelian and non-Abelian gauge transformations.

In our work, in the collinear space, one has $A_{s \mu} \tilde{s}^{i}=$ $\left(\mathbf{h}_{\mathbf{R}} \cdot \tilde{\boldsymbol{\sigma}}, 0\right)$, and in the specific gauge with zero superconducting phase, Eq. (5) is obtained. In the helical space, with $A_{s \mu} \tilde{s}^{i}=\left(h_{0} \tilde{\sigma}_{z},\left(\mathbf{A}_{\mathbf{s}} \cdot \tilde{\boldsymbol{\sigma}}\right) \mathbf{e}_{\mathbf{x}}\right)$, by neglecting second order of the modulation wave vector, Eq. (33) is obtained.

\section{Appendix B: $T$-matrix}

We present the scattering $T$-matrix in this section. From the Lippmann-Schwinger equation: ${ }^{40,92-97}$

$$
T_{\mathbf{k k}^{\prime}}(E)=z_{i} V_{\mathbf{k}-\mathbf{k}^{\prime}} \tau_{3}+\sum_{\mathbf{k}_{\mathbf{1}}} z_{i} V_{\mathbf{k}-\mathbf{k}_{\mathbf{1}}} \tau_{3} G_{\mathbf{k}_{\mathbf{1}}}(E) T_{\mathbf{k}_{\mathbf{1}} \mathbf{k}^{\prime}}
$$

the scattering $T$-matrix is given by

$$
\begin{aligned}
& T_{\mathbf{k k}^{\prime}}(E)=z_{i} V_{\mathbf{k}-\mathbf{k}^{\prime}} \tau_{3}+\sum_{\mathbf{k}_{\mathbf{1}}} z_{i} V_{\mathbf{k}-\mathbf{k}_{\mathbf{1}}} \tau_{3} G_{\mathbf{k}_{\mathbf{1}}}(E) z_{i} V_{\mathbf{k}_{\mathbf{1}}-\mathbf{k}^{\prime}} \tau_{3} \\
& +\sum_{\mathbf{k}_{\mathbf{1}} \mathbf{k}_{\mathbf{2}}} z_{i} V_{\mathbf{k}-\mathbf{k}_{\mathbf{1}}} \tau_{3} G_{\mathbf{k}_{\mathbf{1}}}(E) z_{i} V_{\mathbf{k}_{\mathbf{1}}-\mathbf{k}_{\mathbf{2}}} \tau_{3} G_{\mathbf{k}_{\mathbf{2}}}(E) z_{i} V_{\mathbf{k}_{\mathbf{2}}-\mathbf{k}^{\prime}} \tau_{3} \\
& +\ldots
\end{aligned}
$$

with Green function $G_{\mathbf{k}_{\mathbf{1}}}(E)=\frac{1}{E-H_{0 \mathbf{k}}}=\frac{E-\xi_{k} \tau_{3}-\Delta_{\mathbf{k}}^{\mathbf{R}}\left(\mathbf{d}_{0}\right)}{E^{2}-E_{k}^{2}}$.

It is noted that the impurity interaction $z_{i} V_{\mathbf{k k}^{\prime}}$ has the weak momentum dependence, since the screening constant $\kappa$ in our study is compared to the Fermi vector.
Consequently, Eq. (B2) approximately becomes:

$$
\begin{aligned}
& T_{\mathbf{k k}^{\prime}}(E) \approx z_{i} V_{\mathbf{k}-\mathbf{k}^{\prime}} \tau_{3}+z_{i} V_{\mathbf{k}-\mathbf{k}^{\prime}} \tau_{3}\left[\sum_{\mathbf{k}_{\mathbf{1}}} G_{\mathbf{k}_{\mathbf{1}}}(E)\right] z_{i} V_{0} \tau_{3} \\
& +z_{i} V_{\mathbf{k}-\mathbf{k}^{\prime}} \tau_{3}\left[\sum_{\mathbf{k}_{\mathbf{1}}} G_{\mathbf{k}_{\mathbf{1}}}(E)\right] z_{i} V_{0} \tau_{3}\left[\sum_{\mathbf{k}_{\mathbf{2}}} G_{\mathbf{k}_{\mathbf{2}}}(E)\right] z_{i} V_{\mathbf{0}} \tau_{3} \\
& +\ldots=\frac{z_{i} V_{\mathbf{k}-\mathbf{k}^{\prime}} \tau_{3}}{1+i \tau_{3} \pi \nu z_{i} V_{0} E / \sqrt{E^{2}-\Delta_{0}^{2}}}
\end{aligned}
$$

similar to the previous work for the short-range impurity interaction. ${ }^{40}$ For quasiparticles with energy $E=E_{k}>$ $\Delta_{0}$, the scattering $T$-matrix used in Sec. II is obtained.

\section{Appendix C: Normal quasiparticle current}

In this part, we show that the optically excited normal quasiparticle current always lies in the linear-response (i.e., small- $\left.E_{0}\right)$ regime in our investigation. This can be clearly seen from Fig. 8, where it is shown that the maximum of the excited normal quasiparticle current increases linearly with the electric-field strength.

\section{Appendix D: Derivation of Eq. (34)}

We derive Eq. (34) in this part. By neglecting the effective chemical potential, $\mathrm{HF}$ and impurity scattering terms, in the linear response, we first transform Eq. (33) from the particle space into the quasiparticle one as:

$$
\begin{aligned}
& i \Omega \rho_{\mathbf{k}}^{\tilde{q}}+i\left[\left(E_{k}+h_{0} \tilde{\sigma}_{z}\right) \tau_{3}, \rho_{\mathbf{k}}^{\tilde{q}}\right]+\frac{1}{2}\left\{e E_{0} t_{3},\left[U_{\mathbf{k}}^{\dagger} \partial_{\mathbf{k}} U_{\mathbf{k}}, \rho_{\mathbf{k}}^{\tilde{q}}\right]\right\} \\
& +\frac{1}{2}\left\{\left(\mathbf{c}_{\mathbf{s}} \cdot \tilde{\boldsymbol{\sigma}}\right) t_{3}, \partial_{k_{x}} \rho_{\mathbf{k}}^{\tilde{q}}+\left[U_{\mathbf{k}}^{\dagger} \partial_{\mathbf{k}} U_{\mathbf{k}}, \rho_{\mathbf{k}}^{\tilde{q}}\right]\right\} \\
& -\left\{\frac{i k_{x}}{2 m} t_{3},\left[\left(\mathbf{A}_{\mathbf{s}} \cdot \tilde{\boldsymbol{\sigma}}\right) t_{3}, \rho_{\mathbf{k}}^{\tilde{q}}\right]\right\}=0 .
\end{aligned}
$$

Here, $\left(\mathbf{c}_{\mathbf{s}} \cdot \tilde{\boldsymbol{\sigma}}\right) \tau_{3}=\left[\left(\mathbf{A}_{\mathbf{s}} \cdot \tilde{\boldsymbol{\sigma}}\right), h_{0} \tilde{\sigma}\right]$ and $\rho_{\mathbf{k}}^{\tilde{q}}=U_{\mathbf{k}} \rho_{\mathbf{k}}^{h} U_{\mathbf{k}}$. In Eq. (D1), we have neglected the in-plane triplet vector and the excited effective in-plane magnetic field [seventh term in Eq. [33)] due to the large out-of-plane triplet vector and small $p_{s}$, respectively.

Since the conical angle $\sin (\theta / 2)$ is a small quantity in our work, one can expand the density matrix as $\rho_{\mathbf{k}}^{\tilde{q}}=$ $\sum_{l} \rho_{\mathbf{k}}^{l}$ with $l$ denoting the order of conical angle, and Eq. (D1) becomes:

$$
\begin{aligned}
& \Omega \rho_{\mathbf{k}}^{l}+\left[\left(E_{k}+h_{0} \tilde{\sigma}_{z}\right) \tau_{3}, \rho_{\mathbf{k}}^{l}\right]-\frac{k_{x}}{2 m}\left\{t_{3},\left[\left(\mathbf{A}_{\mathbf{s}} \cdot \tilde{\boldsymbol{\sigma}}\right) t_{3}, \rho_{\mathbf{k}}^{l-1}\right]\right\} \\
& -\frac{i}{2}\left\{\left(\mathbf{c}_{\mathbf{s}} \cdot \tilde{\boldsymbol{\sigma}}\right) t_{3}, \partial_{k_{x}} \rho_{\mathbf{k}}^{l-1}+\left[B_{\mathbf{k}}, \rho_{\mathbf{k}}^{l-1}\right]\right\}=0 .
\end{aligned}
$$

From above equation, one has $\rho_{\mathbf{k}}^{l=0}=-i D_{1} k_{x} \tau_{0}, \rho_{\mathbf{k}}^{l=1}=$ $D_{1}\left(\mathbf{c}_{s} \cdot \tilde{\boldsymbol{\sigma}}\right) t_{\Omega}$ and $\rho_{\mathbf{k}}^{l=2}=D_{2} k_{x} \tilde{\sigma}_{z} \tau_{3} t_{\Omega}$ in the lowest three 
orders. Here, $D_{1}=e E_{0} \frac{2 u_{k}^{2} v_{k}^{2}}{\Omega m E_{k}}, D_{2}=\frac{2 i D_{1} A_{s} c_{s}}{m \Omega}$ and $t_{\Omega}=$ $\frac{u_{k}^{2}-v_{k}^{2}}{\Omega} \tau_{3}+\sum_{ \pm} \frac{2 u_{k} v_{k}}{\Omega \pm 2 E_{k}} \sigma_{1} \tau_{ \pm}$. Then, $\rho_{\mathbf{k}}^{l=3}$ is obtained as

$$
\begin{aligned}
\rho_{\mathbf{k}}^{l=3}= & D_{3}^{0}\left(\begin{array}{cc}
\frac{2 u_{k} v_{k}\left(\mathbf{e}_{\mathbf{z}} \times \mathbf{c}_{\mathbf{s}}\right) \cdot \boldsymbol{\sigma}}{\Omega^{2}-4 E_{k}^{2}} & \frac{\left(v_{k}^{2}-u_{k}^{2}\right)\left(\mathbf{e}_{\mathbf{z}} \times \mathbf{c}_{\mathbf{s}}\right) \cdot \boldsymbol{\sigma} \sigma_{1}}{\left(\Omega+2 E_{k}\right)^{2}} \\
\frac{\left(v_{k}^{2}-u_{k}^{2}\right) \sigma_{1}\left(\mathbf{e}_{\mathbf{z}} \times \mathbf{c}_{\mathbf{s}}\right) \cdot \boldsymbol{\sigma}}{\left(\Omega-2 E_{k}\right)^{2}} & -\frac{2 u_{k} v_{k}\left(\mathbf{e}_{\mathbf{z}} \times \mathbf{c}_{\mathbf{s}}\right) \cdot \boldsymbol{\sigma}^{*}}{\Omega^{2}-4 E_{k}^{2}}
\end{array}\right) \\
& -\frac{i D_{3}^{0} k_{x} k_{y}}{k^{2}}\left(\begin{array}{cc}
\frac{D_{3}^{1}\left(\mathbf{e}_{\mathbf{z}} \times \mathbf{c}_{\mathbf{s}}\right) \cdot \boldsymbol{\sigma}}{\Omega} & \frac{D_{3}^{+}\left(\mathbf{e}_{\mathbf{z}} \times \mathbf{c}_{\mathbf{s}}\right) \cdot \boldsymbol{\sigma} \sigma_{1}}{\Omega+2 E_{k}} \\
-\frac{D_{3}^{-} \sigma_{1}\left(\mathbf{e}_{\mathbf{z}} \times \mathbf{c}_{\mathbf{s}}\right) \cdot \boldsymbol{\sigma}}{\Omega\left(2 E_{k}\right.} & -\frac{D_{3}^{1}\left(\mathbf{e}_{\mathbf{z}} \times \mathbf{c}_{\mathbf{s}}\right) \cdot \boldsymbol{\sigma}^{*}}{\Omega}
\end{array}\right) \\
& +2 i h_{0} D_{3}^{0}\left(\begin{array}{cc}
\frac{2 u_{k} v_{k}\left(\mathbf{c}_{\mathbf{s}} \cdot \boldsymbol{\sigma}\right)}{\Omega\left(\Omega^{2}-4 E_{k}^{2}\right)} & \frac{\left(v_{k}^{2}-u_{k}^{2}\right)\left(\mathbf{c}_{\mathbf{s}} \cdot \boldsymbol{\sigma}\right) \sigma_{1}}{\left(\Omega+2 E_{k}\right)^{3}} \\
\frac{\left(v_{k}^{2}-u_{k}^{2}\right) \sigma_{1}\left(\mathbf{c}_{\mathbf{s}} \cdot \boldsymbol{\sigma}\right)}{\left(\Omega-2 E_{k}\right)^{3}} & -\frac{2 u_{k} v_{k}\left(\mathbf{c}_{\mathbf{s}} \cdot \boldsymbol{\sigma}^{*}\right)}{\Omega\left(\Omega^{2}-4 E_{k}^{2}\right)}
\end{array}\right) \\
& +\frac{2 h_{0} D_{3}^{0} k_{x} k_{y}}{k^{2}}\left(\begin{array}{cc}
\frac{D_{3}^{1}\left(\mathbf{c}_{\mathbf{s}} \cdot \boldsymbol{\sigma}\right)}{\Omega^{2}} & \frac{D_{3}^{+}\left(\mathbf{c}_{\mathbf{s}} \cdot \boldsymbol{\sigma}\right) \sigma_{1}}{\left(\Omega+2 E_{k}\right)^{2}} \\
-\frac{D_{3}^{-} \sigma_{1}\left(\mathbf{c}_{\mathbf{s}} \cdot \boldsymbol{\sigma}\right)}{\left(\Omega-2 E_{k}\right)^{2}} & -\frac{D_{3}^{1}\left(\mathbf{c}_{\mathbf{s}} \cdot \boldsymbol{\sigma}^{*}\right)}{\Omega^{2}}
\end{array}\right),(D)
\end{aligned}
$$

with $D_{3}^{0}=\frac{4 u_{k} v_{k} D_{2} E_{k}}{\Omega}, D_{3}^{1}=\frac{4 u_{k} v_{k} E_{k}\left(u_{k}^{2}-v_{k}^{2}\right)}{\Omega^{2}-4 E_{k}^{2}}$ and $D_{3}^{ \pm}=$ $\frac{4 u_{k}^{2} v_{k}^{2} \Omega}{\Omega^{2}-4 E_{k}^{2}}+\frac{\left(u_{k}^{2}-v_{k}^{2}\right)^{2}}{\Omega \pm 2 E_{k}}$.

Finally, from Eqs. (D2) and (D3), the induced anomalous Hall current reads:

$$
\begin{aligned}
I_{y} & =\frac{e}{2 m} \int d \mathbf{k} \operatorname{Tr}\left(k_{y} \rho_{\mathbf{k}}^{\tilde{q}}\right) \approx \frac{e}{2 m} \int d \mathbf{k} \operatorname{Tr}\left(k_{y} \rho_{\mathbf{k}}^{4}\right) \\
& =\frac{e}{\pi} \int d \varepsilon_{k} D_{4}\left[\frac{2 \xi_{k}}{E_{k}} \frac{D_{3}^{1}}{\Omega^{2}}+\sum_{ \pm} \frac{2 u_{k} v_{k} D_{3}^{ \pm}}{\left(\Omega \pm 2 E_{k}\right)^{2}}\right]
\end{aligned}
$$

which becomes Eq. (34) at high frequency.

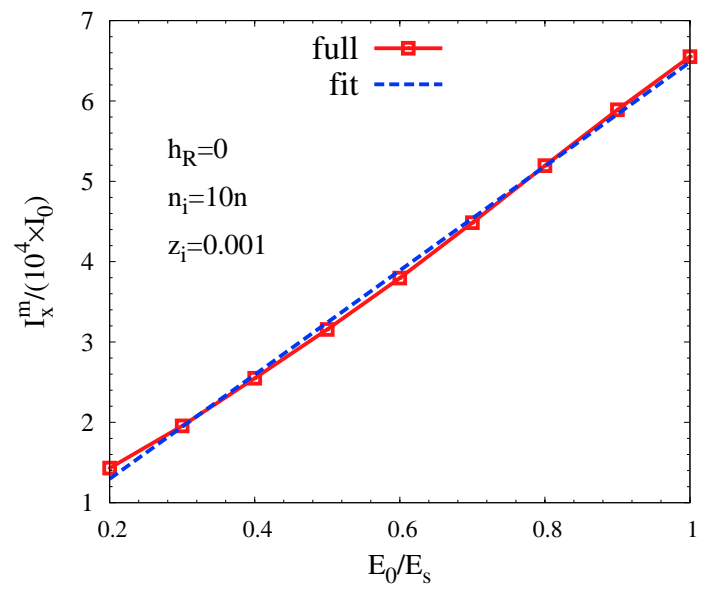

FIG. 8: (Color online) Maximum of the excited normal quasiparticle current versus electric-field strength. Blue dashed curve: fitted result by using $I_{x} \propto E_{0}$.
* Author to whom correspondence should be addressed; Electronic address: mwwu@ustc.edu.cn.

1 Y. Maeno, H. Hashimoto, K. Yoshida, S. Nishizaki, T. Fujita, J. G. Bednorz, and F. Lichtenberg, Nature (London) 372, 532 (1994).

2 A. P. Morenzoni and Y. Maeno, Phys. Res. Sect. B 192, 254 (2002).

3 A. P. Mackenzie and Y. Maeno, Rev. Mod. Phys. 75, 657 (2003).

4 V. P. Mineev, J. Low Temp. Phys. 158, 615 (2010).

5 Y. Maeno, S. Kittaka, T. Nomura, S. Yonezawa, and K. Ishida, J. Phys. Soc. Jpn. 81, 011009 (2012).

${ }^{6}$ C. Kallin, Rep. Prog. Phys. 75, 042501 (2012).

7 C. Kallin and J. Berlinsky, Rep. Prog. Phys. 79, 054502 (2016).

8 J. A. Duffy, S. M. Hayden, Y. Maeno, Z. Mao, J. Kulda, and G. J. McIntyre, Phys. Rev. Lett. 85, 5412 (2000).

9 K. Ishida, H. Mukuda, Y. Kitaoka, K. Asayama, Z. Mao, Y. Mori, and Y. Maeno, Nature (London) 396, 658 (1998).

10 K. Ishida, H. Mukuda, Y. Kitaoka, Z. Q. Mao, H. Fukazawa, and Y. Maeno, Phys. Rev. B 63, 060507 (2001).

11 H. Murakawa, K. Ishida, K. Kitagawa, Z. Q. Mao, and Y. Maeno, Phys. Rev. Lett. 93, 167004 (2004).

12 A. P. Mackenzie, R. K. W. Haselwimmer, A. W. Tyler, G. G. Lonzarich, Y. Mori, S. Nishizaki, and Y. Maeno, Phys. Rev. Lett. 80, 3890 (1998).

13 A. P. Mackenzie, R. K. W. Haselwimmer, A. W. Tyler,
G. G. Lonzarich, Y. Mori, S. Nishizaki, and Y. Maeno, Phys. Rev. Lett. 80, 161 (1998).

14 R. Jin, Y. Liu, Z. Q. Mao, and Y. Maeno, Europhys. Lett. 51, 341 (2000).

${ }^{15}$ K. Nelson, Z. Mao, Y. Maeno, and Y. Liu, Science 306, 1151 (2004).

16 F. Kidwingira, J. D. Strand, D. J. Van Harlingen, and Y. Maeno, Science 314, 1267 (2006).

17 G. M. Luke, Y. Fudamoto, K. M. Kojima, M. I. Larkin, J. Merrin, B. Nachumi, Y. J. Uemura, Y. Maeno, Z. Q. Mao, Y. Mori, H. Nakamura, and M. Sigrist, Nature (London) 394, 558 (1998).

18 J. Xia, Y. Maeno, P. T. Beyersdorf, M. M. Fejer, and A. Kapitulnik, Phys. Rev. Lett. 97, 167002 (2006).

19 M. Sigrist and K. Ueda, Rev. Mod. Phys. 63, 239 (1991).

20 T. Rice and M. Sigrist, J. Phys.: Condens. Matter 7, L643 (1995).

21 G. Baskaran, Physica B 223, 490 (1996).

22 C. Kallin and A. J. Berlinsky, J. Phys.: Condens. Matter 21, 164210 (2009).

23 R. M. Lutchyn, P. Nagornykh, and V. M. Yakovenko, Phys. Rev. B 77, 144516 (2008).

${ }^{24}$ R. Roy and C. Kallin, Phys. Rev. B 77, 174513 (2008).

25 J. Goryo, Phys. Rev. B 78, 060501 (2008).

${ }^{26}$ R. M. Lutchyn, P. Nagornykh, and V. M. Yakovenko, Phys. Rev. B 80, 104508 (2009).

27 E. J. König and A. Levchenko, Phys. Rev. Lett. 118, 027001 (2017).

28 N. Nagaosa, J. Sinova, S. Onoda, A. MacDonald, and N. 
Ong, Rev. Mod. Phys. 82, 1539 (2010).

29 N. Read and D. Green, Phys. Rev. B 61, 10267 (2000).

30 V. Ambegaokar and L. P. Kadanoff, Il Nuovo Cimento 22, 914 (1961).

31 J. R. Schrieffer, Theory of Superconductivity (W. A. Benjamin, New York, 1964).

32 P. W. Anderson, Rev. Mod. Phys. 38, 298 (1966).

33 S. B. Nam, Phys. Rev. 156, 470 (1967); Phys. Rev. B 2, 3812 (1970)

34 C. P. Enz, Rev. Mod. Phys. 46, 705 (1974).

35 R. Coté and A. Griffin, Phys. Rev. B 48, 10404 (1993).

36 S. D. Brorson, R. Buhleier, I. E. Trofimov, J. O. White, C. Ludwig, F. F. Balakirev, H. U. Habermeier, and J. Kuhl, J. Opt. Soc. Am. B 13, 1979 (1996).

37 A. Altland and B. Simons, Condensed Matter Field Theory (Cambridge University Press, Cambridge, England, 2010).

38 Z. Dai and P. A. Lee, Phys. Rev. B 95, 014506 (2017).

39 The experimentally observed Kerr effect was also considered within clean multiband models. ${ }^{113-117}$ Nevertheless, Kallin pointed out ${ }^{6}$ that the multiband model deviates from the present approximation and discussion where it is assumed that the superconductivity in $\mathrm{Sr}_{2} \mathrm{RuO}_{4}$ is predominantly on the $\gamma$ band with weak induced superconductivity on the $\alpha$ and $\beta$ bands.

40 S. Li, A. V. Andreev, and B. Z. Spivak, Phys. Rev. B 92, 100506 (2015).

41 T. Yu and M. W. Wu, Phys. Rev. B 96, 155311 (2017).

42 P. Fulde and R. A. Ferrell, Phys. Rev. 135, 550 (1964).

43 A. I. Larkin and Y. N. Ovchinnikov, Zh. Eksp. Teor. Fiz. 47, 1136 (1964) [Sov. Phys. JETP 20, 762 (1965)].

44 S. Takada and T. Izuyama, Prog. Theor. Phys. 41, 635 (1969).

45 I. Khavkine, H. Y. Kee, and K. Maki, Phys. Rev. B 70 , 184521 (2004).

46 G. Tkachov and V. I. Falko, Phys. Rev. B 69, 092503 (2004).

47 F. Rohlfing, G. Tkachov, F. Otto, K. Richter, D. Weiss, G. Borghs, and C. Strunk, Phys. Rev. B 80, 220507(R) (2009).

48 T. Yu and M. W. Wu, Phys. Rev. B 94, 205305 (2016).

49 F. Yang and M. W. Wu, Phys. Rev. B 95, 075304 (2017).

50 F. Yang and M. W. Wu, arXiv:1704.0040

51 T. Papenkort, V. M. Axt, and T. Kuhn, Phys. Rev. B 76, 224522 (2007).

52 T. Papenkort, T. Kuhn, and V. M. Axt, Phys. Rev. B 78, 132505 (2008).

53 A. F. Kemper, M. A. Sentef, B. Moritz, J. K. Freericks, and T. P. Devereaux, Phys. Rev. B 92, 224517 (2015).

54 N. Tsuji and H. Aoki, Phys. Rev. B 92, 064508 (2015).

55 M. Dzero, M. Khodas, and A. Levchenko, Phys. Rev. B 91, 214505 (2015).

${ }^{56}$ H. Krull, N. Bittner, G. S. Uhrig, D. Manske, and A. P. Schnyder, Nat. Commun. 7, 11921 (2016).

57 M. Lu, H. W. Liu, P. Wang, and X. C. Xie, Phys. Rev. B 93, 064516 (2016).

58 N. Tsuji, Y. Murakami, and H. Aoki, Phys. Rev. B 94, 224519 (2016).

59 Y. Murotani, N. Tsuji, and H. Aoki, Phys. Rev. B 95, $104503(2017)$

60 T. Yu and M. W. Wu, Phys. Rev. B 96, 155312 (2017).

61 Y. Nambu, Phys. Rev. 117, 648 (1960).

62 Y. Nambu, Rev. Mod. Phys. 81, 1015 (2009).

63 Y. Tanaka, Y. Asano, A. A. Golubov, and S. Kashiwaya,
Phys. Rev. B 72, 140503(R) (2005).

${ }^{64}$ G. Annunziata, M. Cuoco, C. Noce, A. Sudbo, and J. Linder, Phys. Rev. B 83, 060508(R) (2011).

65 A. C. Keser, V. Stanev, and V. Galitski, Phys. Rev. B 91, 094518 (2015).

${ }^{66}$ R. Nakagawa, T. Nakamura, T. Terashima, S. Yonezawa, and Y. Maeno, Physica C 470, S744 (2010).

67 T. Nakamura, R. Nakagawa, T. Yamagishi, T. Terashima, S. Yonezawa, M. Sigrist, and Y. Maeno, Phys. Rev. B 84, 060512(R) (2011).

68 T. Nakamura, T. Sumi, S. Yonezawa, T. Terashima, M. Sigrist, H. Kaneyasu, and Y. Maeno, J. Phys. Soc. Jpn. 81, 064708 (2012).

69 M. S. Anwar, S. R. Lee, R. Ishiguro, Y. Sugimoto, Y. Tano, S. J. Kang, Y. J. Shin, S. Yonezawa, D. Manske, H. Takayanagi, T. W. Noh, and Y. Maeno, Nat. Commu. 7, 13220 (2016).

${ }^{70}$ H. Takayanagi and T. Kawakami, Phys. Rev. Lett. 54, 2449 (1985).

71 T. Akazaki, H. Takayanagi, J. Nitta, and T. Enoki, Appl. Phys. Lett. 68, 418 (1996).

${ }^{72}$ K. M. H. Lenssen, M. Matters, C. J. P. M. Harmans, and J. E. Mooji, Appl. Phys. Lett. 63, 2079 (1993).

73 T. D. Moore and D. A. Williams, Phys. Rev. B 59, 7308 (1999).

74 Z. Wan, A. Kazakov, M. J. Manfra, L. N. Pfeiffer, K. W. West, and L. P. Rokhinson, Nat. Commun. 6, 7426 (2015).

75 Semiconductors, Landolt-Börnstein, New Series, Vol. 17a, edited by O. Madelung (Springer, Berlin, 1987).

76 I. Sosnowska, T. P. Neumaier, and E. Steichele, J. Phys. C: Solid State Phys. 15, 4835 (1982).

77 T. H. Arima, J. Phys. Soc. Jpn. 76, 073702 (2007).

78 T. Kimura and Y. Tokura, J. Phys.: Condens. Matter 20, 434204 (2008).

79 X. Ke, P. P. Zhang, S. H. Baek, J. Zarestky, W. Tian, and C. B. Eom, Phys. Rev. B 82, 134448 (2010).

${ }^{80}$ C. T. Wu, O. T. Valls, and K. Halterman, Phys. Rev. Lett. 108, 117005 (2012).

81 A. J. Leggett, Quantum Liquids (Oxford University Press, Oxford, 2006).

${ }^{82}$ L. P. Gor'kov and G. M. Eliashberg, Zh. Eksp. Teor. Fiz. 51, 612 (1968) [Sov. Phys. JETP 27, 328 (1968)].

83 M. Levanda and V. Fleurov, J. Phys.: Condens. Matter 6, 7889 (1994).

84 M. E. Peskin and D. V. Schroeder, An Introduction to Quantum Field Theory (Addison-Wesley, New York, 1995).

${ }^{85}$ H. Haug and A. P. Jauho, Quantum Kinetics in Transport and Optics of Semiconductors (Springer, Berlin, 1996).

86 T. Kita, Phys. Rev. B 64, 054503 (2001).

87 M. W. Wu, J. H. Jiang, and M. Q. Weng, Phys. Rep. 493, 61 (2010).

88 M. J. Stephen, Phys. Rev. 139, A197 (1965).

89 J. Clarke and M. Tinkham, Phys. Rev. Lett. 44, 106 (1980).

90 H. L. Zhao and S. Hershfield, Phys. Rev. B 52, 3632 (1995).

91 A. G. Aronov, Yu. M. Galperin, V. L. Gurevich, and V. I. Kozub, Nonequilibrium Superconductivity, edited by D. N. Langenberg and A. I. Larkin (Elsevier, New York, 1986).

92 P. Coleman, P. W. Anderson, and T. V. Ramakrishnan, Phys. Rev. Lett. 55, 414 (1985).

93 A. Fert and P. M. Levy, Phys. Rev. B 36, 1907 (1987). 
94 S. Onoda, N. Sugimoto, and N. Nagaosa, Phys. Rev. Lett. 97, 126602 (2006).

95 N. A. Sinitsyn, A. H. MacDonald, T. Jungwirth, V. K. Dugaev, and J. Sinova, Phys. Rev. B 75, 045315 (2007).

96 A. Ferreira, T. G. Rappoport, M. A. Cazalilla, and A. H. C. Neto, Phys. Rev. Lett. 112, 066601 (2014).

97 M. Milletari and A. Ferreira, Phys. Rev. B 94, 134202 (2016).

98 M. Beck, M. Klammer, S. Lang, P. Leiderer, V. V. Kabanov, G. N. Gol'tsman, and J. Demsar, Phys. Rev. Lett. 107, 177007 (2011).

99 R. Matsunaga and R. Shimano, Phys. Rev. Lett. 109, 187002 (2012).

100 R. Matsunaga, Y. I. Hamada, K. Makise, Y. Uzawa, H. Terai, Z. Wang, and R. Shimano, Phys. Rev. Lett. 111, 057002 (2013).

101 R. Matsunaga, N. Tsuji, H. Fujita, A. Sugioka, K. Makise, Y. Uzawa, H. Terai, Z. Wang, H. Aoki, and R. Shimano, Science 345, 1145 (2014).

102 R. Matsunaga, N. Tsuji, K. Makise, H. Terai, H. Aoki, and R. Shimano, Phys. Rev. B 96, 020505 (2017).

103 C. Jia and J. Berakdar, Phys. Rev. B 80, 014432 (2009).

104 C. Jia and J. Berakdar, Appl. Phys. Lett. 95, 012105 (2009).

105 P. Zhang and M. W. Wu, Phys. Rev. B 84, 014433 (2011).

106 For $s$-wave case, we also find that the drive effect dominates the optically excited effective chemical potential in the weak impurity interaction (not shown), which is absent in the previous work ${ }^{41}$ This is in marked contrast to the conclusions from the Liouville equation ${ }^{51-53}$ or Bloch equation ${ }^{54-59}$ in the literature, in which the drive effect is overlooked.

107 A. F. Volkov, F. S. Bergeret, and K. B. Efetov, Phys. Rev. Lett. 90, 117006 (2003).

108 F. S. Bergeret, A. F. Volkov, and K. B. Efetov, Rev. Mod. Phys. 77, 1321 (2005).

109 K. Halterman, P. H. Barsic, and O. T. Valls, Phys. Rev. Lett. 99, 127002 (2007).

110 T. S. Khaire, M. A. Khasawneh, W. P. Pratt, Jr., and N. O. Birge, Phys. Rev. Lett. 104, 137002 (2010).

111 H. Meng, X. Q. Wu, and Z. M. Zheng, Europhys. Lett. 104, 37003 (2013).

112 S. Hikino and S. Yunoki, Phys. Rev. B 92, 024512 (2015).

113 K. I. Wysokiński, J. F. Annett, and B. L. Györffy, Phys. Rev. Lett. 108, 077004 (2012).

114 V. P. Mineev, J. Phys. Soc. Jpn. 81, 093703 (2012).

115 E. Taylor and C. Kallin, Phys. Rev. Lett. 108, 157001 (2012).

116 E. Taylor and C. Kallin, J. Phys. Conf. Ser. 449, 012036 (2013).

117 M. Gradhand, K. I. Wysokinski, J. F. Annett, and B. L. Györffy, Phys. Rev. B 88, 094504 (2013). 